\title{
Small-molecule activator of glutamate transporter EAAT2 translation provides neuroprotection
}

\author{
Qiongman Kong, ${ }^{1}$ Ling-Chu Chang, ${ }^{1}$ Kou Takahashi, ${ }^{1}$ Qibing Liu, ${ }^{1}$ Delanie A. Schulte, ${ }^{1}$ Liching Lai, ${ }^{1}$ \\ Brian Ibabao, ${ }^{1}$ Yuchen Lin, ${ }^{1}$ Nathan Stouffer, ${ }^{1}$ Chitra Das Mukhopadhyay, ${ }^{1}$ Xuechao Xing, ${ }^{2}$ \\ Kathleen I. Seyb, ${ }^{2}$ Gregory D. Cuny, ${ }^{2,3}$ Marcie A. Glicksman, ${ }^{2}$ and Chien-Liang Glenn Lin'

\begin{abstract}
1Department of Neuroscience, The Ohio State University, Columbus, Ohio, USA. ${ }^{2}$ Laboratory for Drug Discovery in Neurodegeneration, Harvard NeuroDiscovery Center, Brigham and Women's Hospital and Harvard Medical School, Cambridge, Massachusetts, USA. 3Department of Pharmacological and Pharmaceutical Sciences, University of Houston, College of Pharmacy, Houston, Texas, USA.
\end{abstract}

\begin{abstract}
Glial glutamate transporter EAAT2 plays a major role in glutamate clearance in synaptic clefts. Several lines of evidence indicate that strategies designed to increase EAAT2 expression have potential for preventing excitotoxicity, which contributes to neuronal injury and death in neurodegenerative diseases. We previously discovered several classes of compounds that can increase EAAT2 expression through translational activation. Here, we present efficacy studies of the compound LDN/OSU-0212320, which is a pyridazine derivative from one of our lead series. In a murine model, LDN/OSU-0212320 had good potency, adequate pharmacokinetic properties, no observed toxicity at the doses examined, and low side effect/toxicity potential. Additionally, LDN/OSU-0212320 protected cultured neurons from glutamate-mediated excitotoxic injury and death via EAAT2 activation. Importantly, LDN/OSU-0212320 markedly delayed motor function decline and extended lifespan in an animal model of amyotrophic lateral sclerosis (ALS). We also found that LDN/OSU-0212320 substantially reduced mortality, neuronal death, and spontaneous recurrent seizures in a pilocarpine-induced temporal lobe epilepsy model. Moreover, our study demonstrated that LDN/OSU-0212320 treatment results in activation of PKC and subsequent Y-box-binding protein 1 (YB-1) activation, which regulates activation of EAAT2 translation. Our data indicate that the use of small molecules to enhance EAAT2 translation may be a therapeutic strategy for the treatment of neurodegenerative diseases.
\end{abstract}

\section{Introduction}

Glutamate is the main excitatory neurotransmitter in the CNS. The concentration of glutamate in the synaptic cleft is tightly regulated by the interplay between glutamate release and glutamate clearance. Under disease conditions, abnormal glutamate release and/or dysfunction of glutamate clearance causes elevated extracellular glutamate concentrations. This results in overstimulation of glutamate receptors, leading to neuronal injury or death, known as excitotoxicity (1), which has been implicated in a wide range of acute and chronic neurodegenerative diseases.

The glial glutamate transporter EAAT2 is found primarily on perisynaptic processes of astrocytes closely associated with excitatory synaptic contacts and is responsible for maintaining low extracellular glutamate concentrations (2-4). Loss of EAAT2 protein and function is commonly found in chronic neurodegenerative diseases such as amyotrophic lateral sclerosis (ALS) and Alzheimer's disease (AD) (5-7) and may be the main cause of excitotoxicity in these diseases. Restoration of EAAT2 protein levels and function may provide therapeutic benefit for these chronic diseases. In disease conditions such as epilepsy, stroke, and neurotrauma, an acute, dramatic increase in extracellular glutamate levels causes severe neuronal damage (8-10). Immediate upregulation of EAAT2 protein will reduce extracellular glutamate levels and thereby prevent damage to neurons. Therefore, increased EAAT2 expression is a potential therapeu-

Conflict of interest: The authors have declared that no conflict of interest exists. Citation for this article: J Clin Invest. 2014;124(3):1255-1267. doi:10.1172/JCI66163. tic approach for preventing excitotoxicity for both chronic and acute neurodegenerative diseases.

EAAT2 can be upregulated by transcriptional (11-13) or translational (14) activation. Rothstein and colleagues discovered that many $\beta$-lactam antibiotics, such as ceftriaxone, are able to increase EAAT2 protein levels through transcriptional activation (15). Our laboratories previously executed high-throughput screenings to search for compounds that increase EAAT2 translation (16). We focused on translational activation, because (a) loss of EAAT2 protein in ALS or $\mathrm{AD}$ patients is most likely due to disturbances at the post-transcriptional level, since EAAT2 mRNA is not decreased $(6,17)$; (b) greater selectivity may be achieved; and (c) immediately increasing EAAT2 protein and function can be achieved, which is critical for an effective therapeutic response. This screening campaign resulted in the discovery of sixteen classes of compounds that can activate EAAT2 translation. After intensive studies of these compounds, a pyridazine-based series was selected for further investigation, including a structure-activity relationship (SAR) study (18). Here, we report characterization and efficacy studies of a representative compound, LDN/OSU-0212320 (Figure 1A), from this lead series.

\section{Results}

LDN/OSU-0212320 increases EAAT2 expression through translational activation in a primary astrocyte cell line. PA-EAAT2 is a rat primary astrocyte line that does not express endogenous EAAT2 protein, but stably expresses human EAAT2 mRNAs driven by the CMV promoter (14). In the absence of serum, PA-EAAT2 cells express low amounts of EAAT2 protein, although the recombinant EAAT2 mRNAs are 


\section{A}

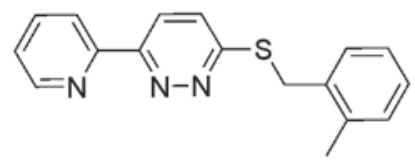

LDN/OSU-0212320

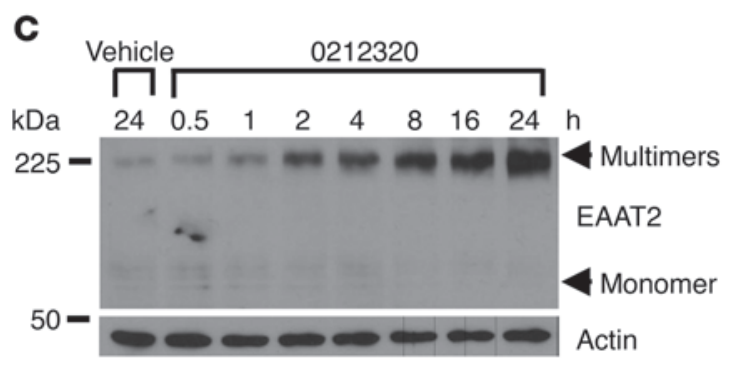

B

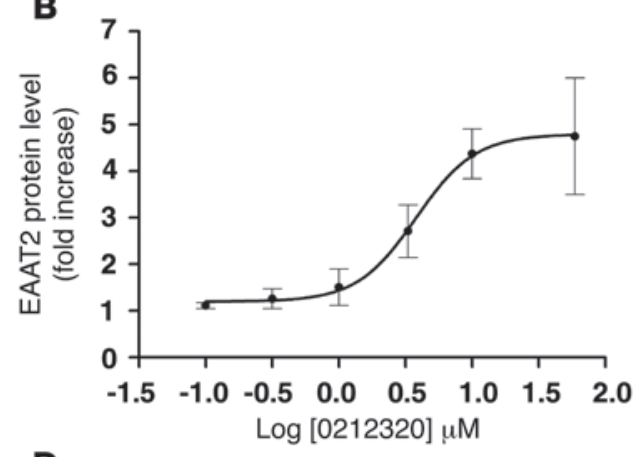

。

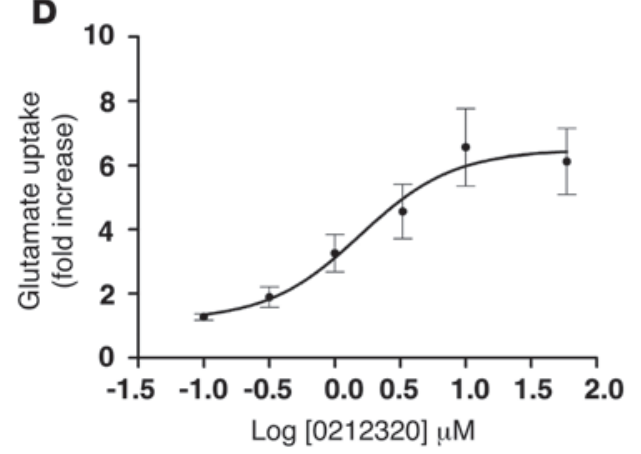

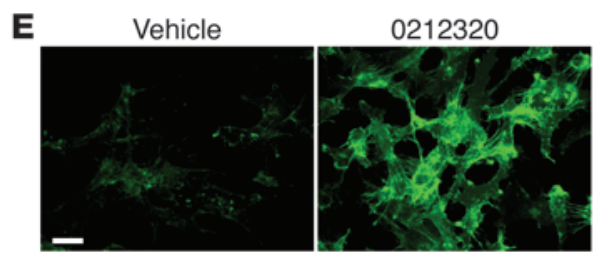
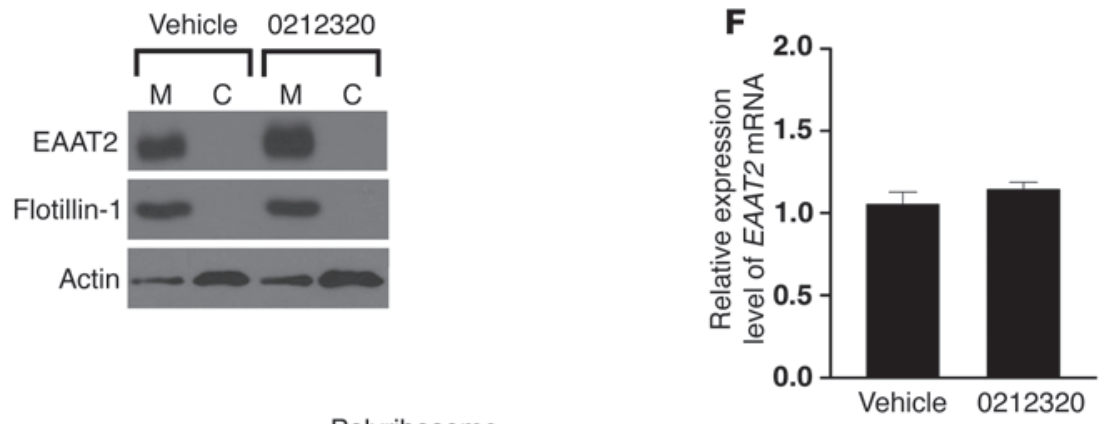

G
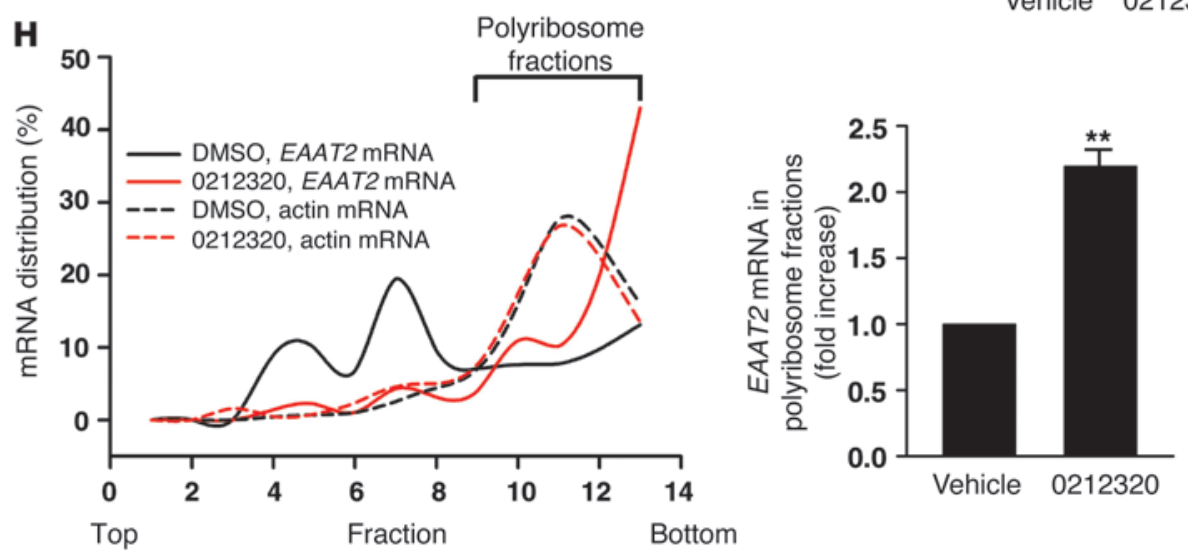


\section{Figure 1}

Characterization of LDN/OSU-0212320 in PA-EAAT2 cells. (A) Structure of LDN/OSU-0212320. (B) Dose response experiments. Cells were treated with compound for 24 hours. $\mathrm{EC}_{50}=1.83 \pm 0.27 \mu \mathrm{M} . n=5$. (C) Time course experiments. Cells were treated with compound at $10 \mu \mathrm{M}$. Compound increased EAAT2 protein levels in a short amount of time. (D) $\left[{ }^{3} \mathrm{H}\right]$ glutamate uptake experiments. Glutamate uptake activity correlated with increased EAAT2 protein levels. Cells were treated with compound for 24 hours. $n=6$. (E) Immunofluorescence staining and subcellular fractionation analysis. Induced EAAT2 was properly localized in the plasma membrane. Cells were treated with compound at a concentration of $3.3 \mu \mathrm{M}$ for 24 hours. M, plasma membrane fraction. C, cytosolic fraction. Scale bar: $25 \mu \mathrm{m}$. (F) Real-time RT-PCR analysis. Compound treatment did not increase EAAT2 mRNA levels. Cells were treated with compound at a concentration of $10 \mu \mathrm{M}$ for $6,18,24$, and 48 hours. Results after 24 hours are shown. $n=5$. (G) Pulse-chase analysis. Compound treatment did not affect the rate of EAAT2 protein degradation. Cells were preincubated with sulfo-NHS-SS-biotin to label surface EAAT2 proteins and were then treated with compound (10 $\mu \mathrm{M})$. Cells were harvested at 8 and 24 hours to measure biotin-labeled EAAT2 protein levels. Equal protein loading was confirmed by Ponceau S staining. (H) Polyribosome analysis. Compound treatment increased EAAT2 mRNA translation activity. Cells were treated with compound at a concentration of $10 \mu \mathrm{M}$ for 1 hour. Cell lysates were prepared and fractionated by a $15 \%$ to $60 \%$ sucrose gradient. RNAs were extracted from each fraction and analyzed by real-time RT-PCR. $n=4 .{ }^{* \star} P<0.01$.

expressed (translational silencing); however, EAAT2 protein levels are increased when an activator is added to the culture (translational activation). We previously used this cell line for high-throughput screening to identify EAAT2 translational activators (16). To prioritize candidate compounds, we initially evaluated the activation of EAAT2 protein in PA-EAAT2 cells. Promising compounds were further evaluated in primary dissociated neuron and astrocyte mixed cultures and then in mice. Figure 1 presents the characterization of LDN/OSU-0212320 in PA-EAAT2 cells. LDN/OSU-0212320 increased EAAT2 protein levels in a dose-dependent (Figure 1B; $\mathrm{EC}_{50}=1.83 \pm 0.27 \mu \mathrm{M}$ ) and time-dependent (Figure 1C) manner. Importantly, the induction occurred in a relatively short amount of time (within 2 hours) and was sustained even up to 72 hours (data not show). The induction was blocked by cycloheximide, an inhibitor of protein biosynthesis (data not shown). A $\left[{ }^{3} \mathrm{H}\right]$ glutamate uptake assay demonstrated that glutamate uptake function correlated with increased EAAT2 protein levels (Figure 1D). Immunofluorescence staining and subcellular fractionation studies showed that induced EAAT2 protein was properly localized in the plasma membrane (Figure 1E). Quantitative real-time RT-PCR analysis revealed that EAAT2 mRNA levels were not changed by LDN/OSU-0212320 treatment (Figure 1F). Pulse-chase experiments indicated that the rate of EAAT2 protein degradation was also not changed by compound treatment (Figure 1G). Polyribosome analysis showed that a large portion of EAAT2 mRNAs shifted from the translational silenced state (nonpolyribosomal fractions; black solid line in Figure $1 \mathrm{H}$ ) to the actively translating state (polyribosomal fractions; red solid line in Figure 1H) 1 hour after adding LDN/OSU-0212320 to cultures, but the compound did not affect actin mRNA translation activity (dotted line in Figure $1 \mathrm{H}$ ). Collectively, these data indicate that LDN/OSU-0212320 increased EAAT2 expression through translational activation.

LDN/OSU-0212320 protects cultured neurons from glutamate-mediated excitotoxic injury/death. We next evaluated LDN/OSU-0212320 in primary dissociated neuron and astrocyte mixed cultures. As shown in Figure 2, A and B, LDN/OSU-0212320 increased EAAT2 protein levels and glutamate uptake function, but did not affect EAAT1 or EAAT3 protein levels (data not shown). Significantly, when the cultures were treated with glutamate for 2 hours to induce excitotoxicity, the compound-treated cultures were protected. Figure $2 \mathrm{C}$ shows that glutamate-induced cytotoxicity, as assessed by lactate dehydrogenase (LDH) assay, was significantly reduced in compound-treated cultures. Figure 2D shows that LDN/OSU-0212320 treatment markedly prevented neuronal loss and degeneration, as assessed by MAP2 immunostaining. On average, $14.6 \pm 4.4 \%$ of MAP2-positive neurons remained after $0.1 \mathrm{mM}$ glutamate treatment when compared with the control, while pretreatment with compound at 2 or $3 \mu \mathrm{M}$ significantly recovered this ratio to $40.4 \pm 6.4 \%$ and $60.9 \pm 7.3 \%$, respectively (Figure $2 \mathrm{E}$ ). These protective effects were partially abolished by pretreatment with dihydrokainic acid (DHK), an EAAT2 blocker, suggesting that activation of EAAT2 is involved in LDN/ OSU-0212320-mediated neuroprotection. Figure 2F shows MAP2 immunolabeling and nuclear condensation (arrows) at a higher magnification. Furthermore, in all assays, LDN/OSU-0212320 did not cause detectable toxicity. It is notable that similar protective effects were also observed in our previous EAAT2 transgenic mice study (19). The study found that primary dissociated cultures derived from EAAT2 transgenic mice could tolerate glutamate toxicity much better than cultures derived from nontransgenic mice. These studies indicate that increased expression of EAAT2 protects cells from glutamate-induced toxicity.

LDN/OSU-0212320 has adequate pharmacokinetic properties, good potency, and no observed toxicity in vivo. LDN/OSU-0212320 is compliant with Lipinski rules and lacks functionality that would predict overt toxicity or reactivity. This compound has a low molecular weight of 293.38, a calculated logarithm of the octanol/water partition coefficient (cLogP) value of 3.28, and a polar surface area (PSA) value of $37.08 \AA^{2}$ in the range typical of compounds that can penetrate the blood-brain barrier. However, the shortcomings of LDN/ OSU-0212320 include poor aqueous solubility (12 $\mu \mathrm{M}, \mathrm{pH} 7.4)$ and a short half-life in mouse liver microsomes ( $\mathrm{t}_{1 / 2}=7$ minutes). We conducted in vivo pharmacokinetic evaluation of LDN/OSU0212320 in male C57Bl/6 mice. After a single i.p. administration of LDN/OSU-0212320 (3 mg/kg), we determined the plasma and brain concentrations by liquid chromatography-tandem mass spectrometry (LC-MS/MS) over an 8-hour period (Figure 3A). LDN/OSU-0212320 reached an average maximum plasma concentration $\left(\mathrm{C}_{\max }\right)$ of $42.1 \pm 3.6 \mathrm{ng} / \mathrm{ml} 15$ minutes after injection. We estimated the plasma and brain half-lives to be 2.63 and 2.64 hours, respectively. The average brain/plasma ratio was approximately 2 at all time points tested. These results indicate that LDN/OSU0212320 has adequate pharmacokinetic properties.

We determined the maximum tolerated dose (MTD) of LDN/ OSU-0212320 in male C57BL/6 mice. The study was conducted in two phases: (a) a single-dose tolerability study tested at doses of $10,25,50$, and $75 \mathrm{mg} / \mathrm{kg}$ i.p. (3 mice/dose); and (b) a 5-day repeatdose tolerability study tested at doses of $10,25,50$, and $100 \mathrm{mg} / \mathrm{kg}$ i.p. (5 mice/dose). All animals were observed for clinical signs of toxicity each day of dosing. In the repeat-dose study, we determined body weights prior to each dose and prior to termination on day 6 . We evaluated clinical pathologies including standard hematology, serum chemistry, and coagulation parameters in samples collected on day 6. All animals from phase II were necropsied, and selected organs were weighed. We observed no clinical signs of toxicity, adverse clinical pathological changes, or body weight changes rel- 
A

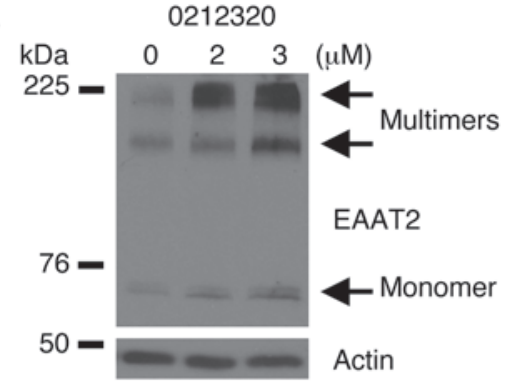

D

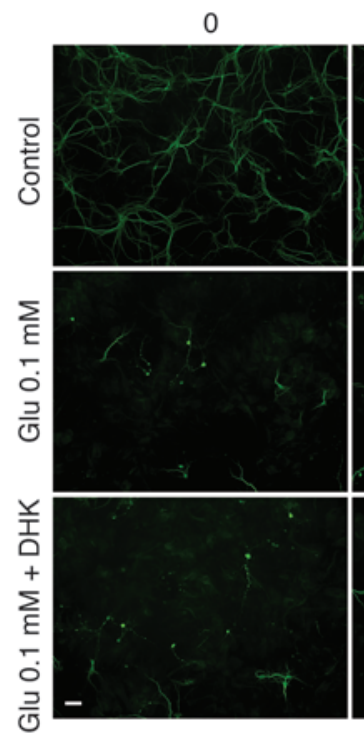

B

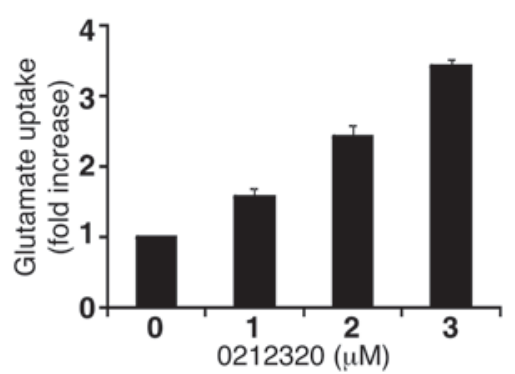

C

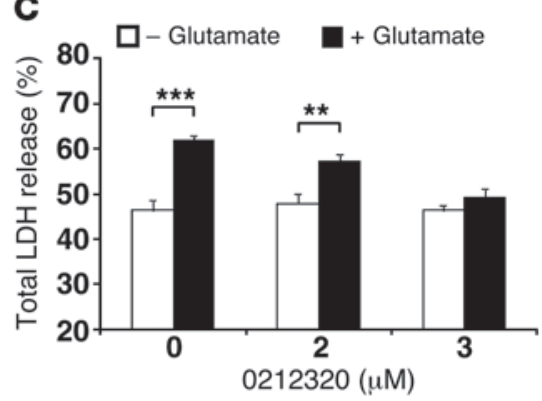

E

$0212320(\mu \mathrm{M})$ 2

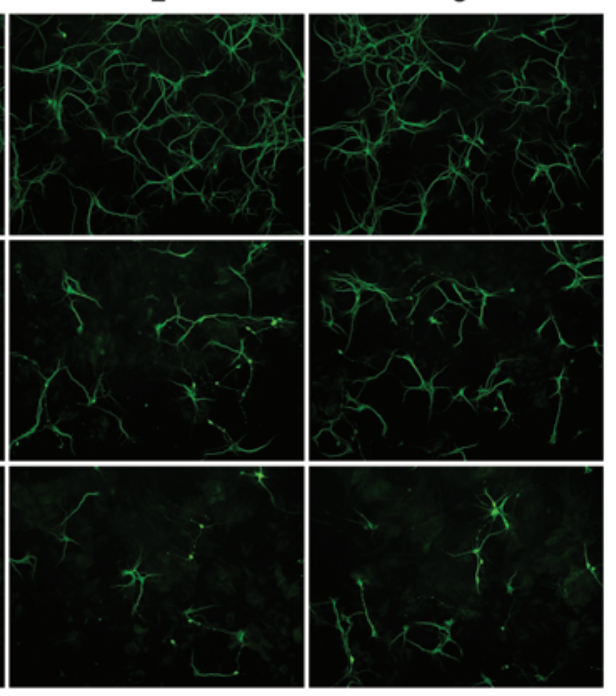

口 $0 \mu \mathrm{M} \quad \square_{2 \mu \mathrm{M}} \quad \square_{3} \mu \mathrm{M} 0212320$

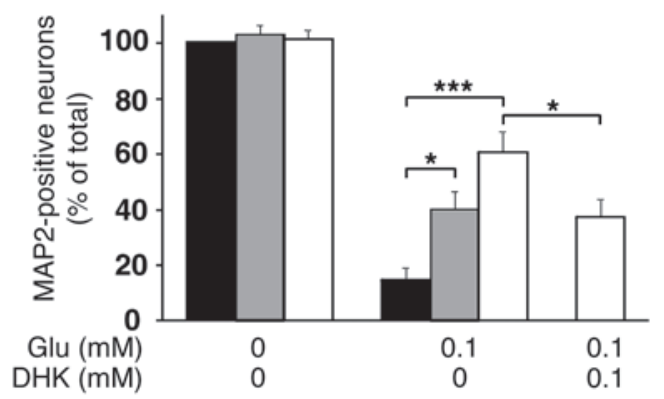

F

Control

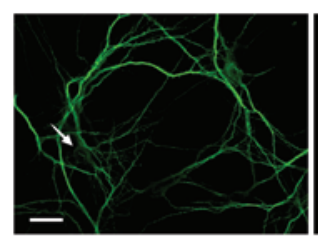

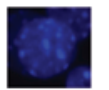

Glu 0.1 mM

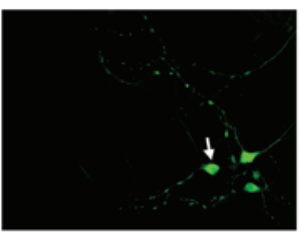

$02123203 \mu \mathrm{M}$ + Glu $0.1 \mathrm{mM}$

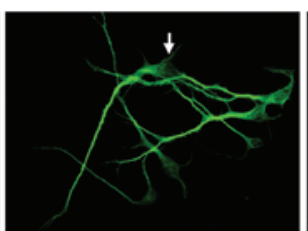

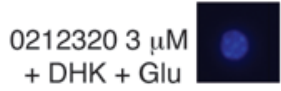

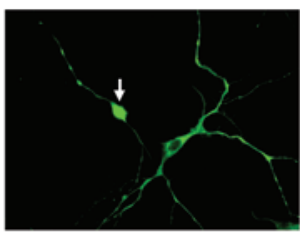

Figure 2

Characterization of LDN/OSU-0212320 in primary dissociated neuron and astrocyte mixed cultures. Seven-day-old primary cultures were treated with the indicated doses of compound for 24 hours and then harvested for analyses. (A) Western blot analysis shows that compound increased EAAT2 expression in a dose-dependent manner. $(B)\left[{ }^{3} \mathrm{H}\right]$ glutamate uptake assay shows a dose-dependently increased glutamate uptake. $n=6$. (C) Cultures were insulted with $0.5 \mathrm{mM}$ glutamate for 2 hours to induce excitotoxicity. LDH assay shows a dose-dependent reduction of LDH release in compound-treated cultures. $n=6$. (D) MAP2 immunostaining shows that compound treatment significantly prevented glutamatemediated neuronal loss and degeneration. The protections were partially abolished by pretreatment of DHK (100 $\mu \mathrm{M})$. Cultures were exposed to $0.1 \mathrm{mM}$ glutamate for 2 hours. Scale bar: $50 \mu \mathrm{m}$. (E) Quantitative analysis of MAP2-positive neurons. MAP2-positive neurons were counted and quantified as described in the Methods. $n=15$. (F) MAP2-immunolabeled neurons and their nuclear morphology (Hoechst 33342 staining) at a higher magnification. Arrows point to the corresponding neurons of the highlighted nuclei. Scale bar: $25 \mu \mathrm{m}$. ${ }^{\star} P<0.05 ;{ }^{* \star} P<0.01 ;{ }^{* \star \star} P<0.001$.

ative to controls at the end of dosing. Due to a lack of toxicity, the highest dose tested, $100 \mathrm{mg} / \mathrm{kg} /$ day, was determined to be the 5 -day no observed adverse effect level (NOAEL) for LDN/OSU-0212320.

We next examined EAAT2 protein levels and glutamate uptake activities in brain following i.p. administration of LDN/OSU0212320 to C57BL/6 mice. Plasma membrane vesicles were prepared from mouse forebrains to measure functional EAAT2 protein. After a single i.p. 40-mg/kg dose of LDN/OSU-0212320,
EAAT2 protein levels and associated glutamate uptake increased by approximately 1.5 - to 2 -fold at 2 hours and by approximately 2 to 3 -fold between 8 and 24 hours after injection (Figure 3B). Even 72 hours after injection, an approximately 1.5-fold increase in EAAT2 protein levels could still be detected (data not shown). In addition, we found that LDN/OSU-0212320-induced EAAT2 protein levels and glutamate uptake were dose dependent (Figure 3C). This induction was not due to transcriptional activation, because Eaat $2 \mathrm{mRNA}$ 
A

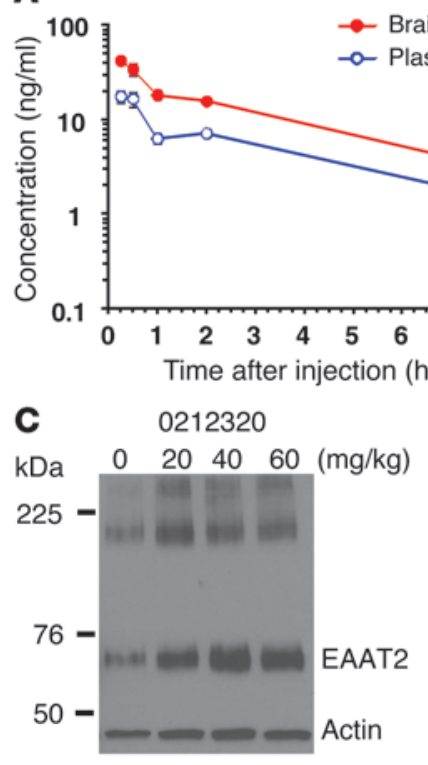

B

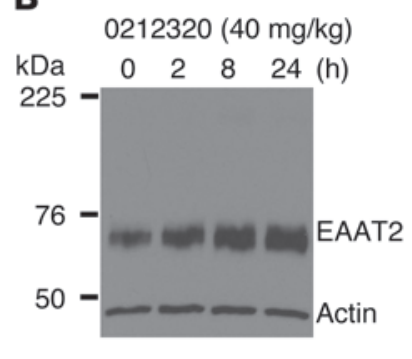

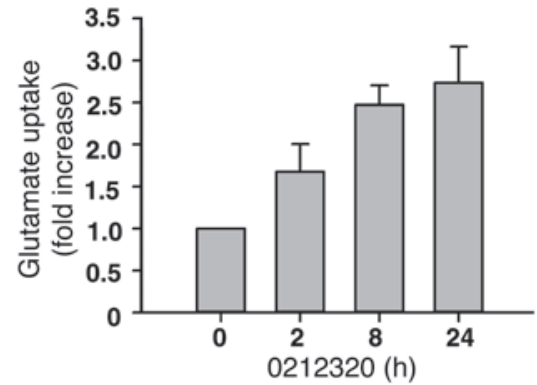
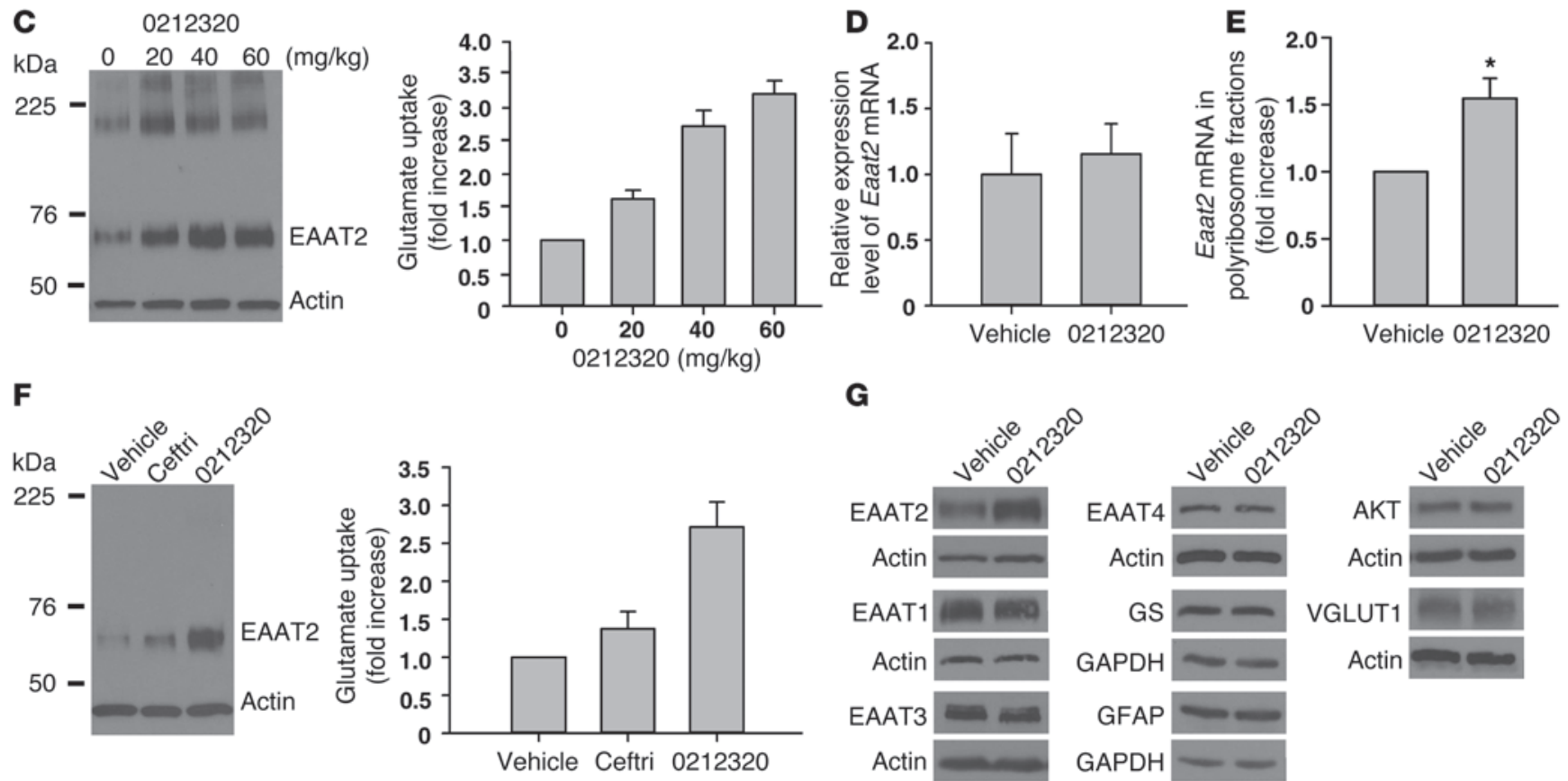

G
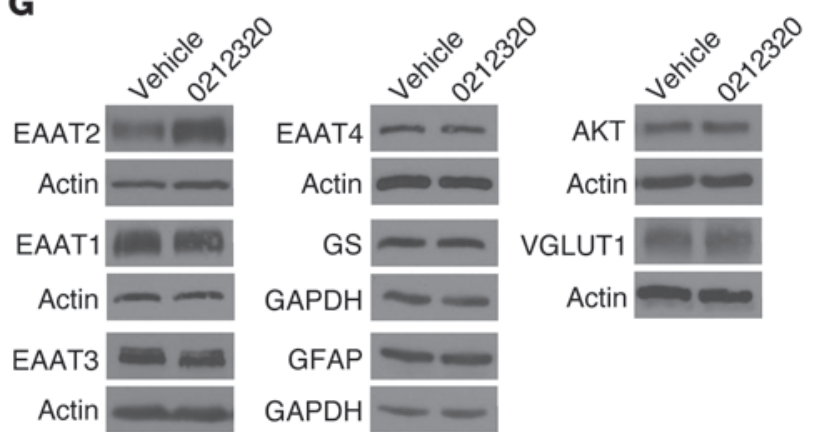

Figure 3

Evaluation of LDN/OSU-0212320 in WT mice. (A) Pharmacokinetic studies. After administration of a single i.p. dose of compound (3 mg/kg) to mice, plasma and brain concentrations were determined ( $n=3 /$ time point). $C_{\max }=42.1 \pm 3.6 \mathrm{ng} / \mathrm{l}$ at 15 minutes, plasma $\mathrm{t}_{1 / 2}=2.63 \mathrm{hours}$, brain $\mathrm{t}_{1 / 2}=2.64$ hours. (B) Time course studies. After a single dose of compound (40 mg/kg), the brains were harvested at the indicated times $(n=3 / \mathrm{time}$ point). Plasma membrane vesicles were prepared from forebrains to determine EAAT2 protein levels and measure [ $\left.{ }^{3} \mathrm{H}\right]$ glutamate uptake activity. Increased EAAT2 could be detected as early as 2 hours after injection. (C) Dose response studies. Mice received a single dose of compound ( $n=3 /$ dose), and brains were harvested at 24 hours after injection. Compound dose-dependently increased EAAT2 levels and glutamate uptake. (D) Quantitative real-time RT-PCR analysis. Eaat2 mRNA levels were not changed by compound treatment. Mice received compound (40 mg/kg), and brains were harvested at 8 and 24 hours. Results at 8 hours are shown. $n=5$. (E) Polyribosome analysis. Compound treatment increased Eaat2 mRNA translation activity. Mice received compound ( $40 \mathrm{mg} / \mathrm{kg})$, and brains were harvested at 1 hour. $n=4$. ( $F)$ Comparison of ceftriaxone (Ceftri) and LDN/OSU-0212320. Mice received a single dose of ceftriaxone (200 mg/kg) or LDN/OSU-0212320 (40 mg/kg) $(n=3 \mathrm{each})$, and brains were harvested at 24 hours. LDN/OSU-0212320 is more potent than ceftriaxone. (G) Western blot analysis of other protein expression levels in LDN/OSU-0212320-treated (40 mg/kg) brains. LDN/OSU-0212320 did not induce global protein synthesis. ${ }^{*} P<0.05$.

was not increased, as measured by quantitative real-time RT-PCR (Figure 3D). Polyribosomal analysis indicated that LDN/OSU0212320 increased Eaat 2 mRNA translation activity (Figure 3E), but did not affect actin mRNA translation activity (data not shown). We compared ceftriaxone $(200 \mathrm{mg} / \mathrm{kg})$, which increases EAAT2 expression through transcriptional activation $(13,15)$, with LDN/ OSU-0212320 (40 mg/kg), which activates EAAT2 translation. As shown in Figure 3F, LDN/OSU-0212320 had greater EAAT2 induction than did ceftriaxone (a single dose after 24 hours). These results indicate that LDN/OSU-0212320 has good potency in vivo.

To assess the biological activity and selectivity of LDN/OSU0212320, we performed an in vitro side-effect profiling study. We tested 71 targets at $10 \mu \mathrm{M}$ in duplicate. These targets included 30 neurotransmitter-related receptors, 2 steroids, 6 ion channels, 1 second messenger, 3 prostaglandins, 4 growth factors/hormones, 13 brain/gut peptides, 5 enzymes, and 7 cytochrome p450. The results showed that LDN/OSU- 0212320 had $69 \%$ inhibition on the cytochrome p450 CYP1A2 and 43\% inhibition on calcium channel L-type at $10 \mu \mathrm{M}$. All other targets demonstrated less than $30 \%$ inhibition at $10 \mu \mathrm{M}$ (Supplemental Table 1; supplemental material available online with this article; doi:10.1172/JCI66163DS1). Additionally, we performed a protein kinase-profiling study. We tested LDN/OSU-0212320 at 1 and $10 \mu \mathrm{M}$ against 230 kinases. The results showed that EPHA4 and EPHA5 were inhibited by $44 \%$ at $10 \mu \mathrm{M}$. 
A
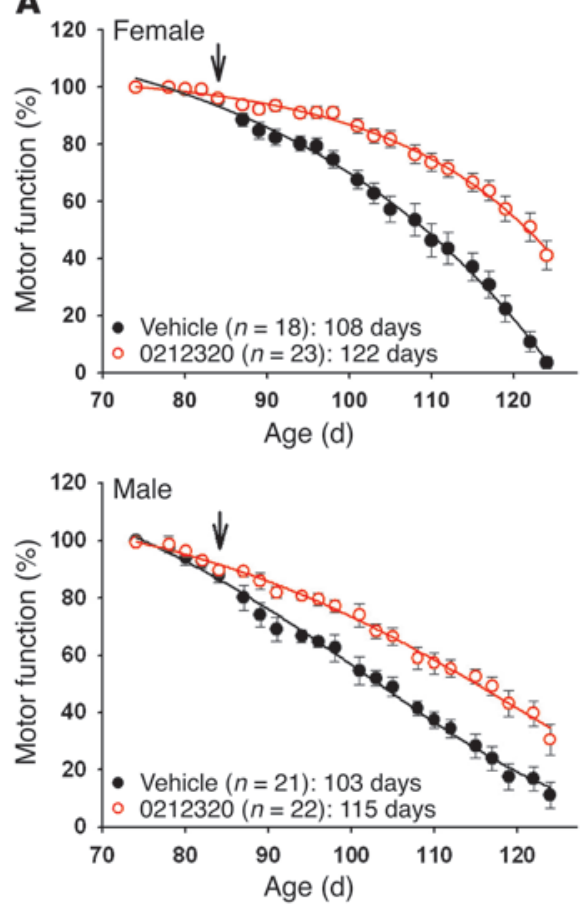

D

At the age of 126 days (females)

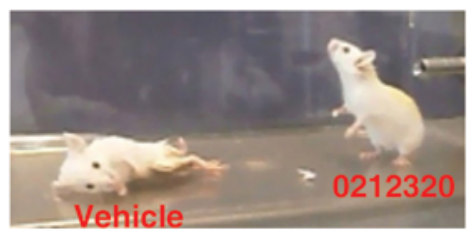

F

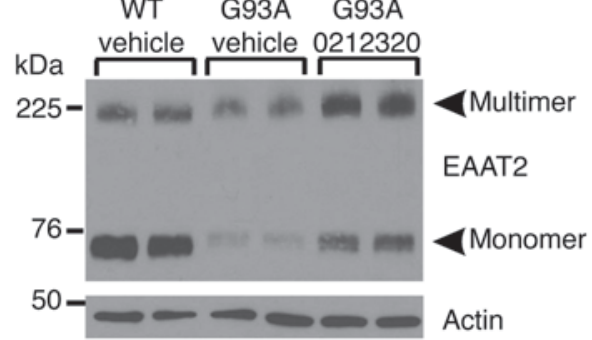

B
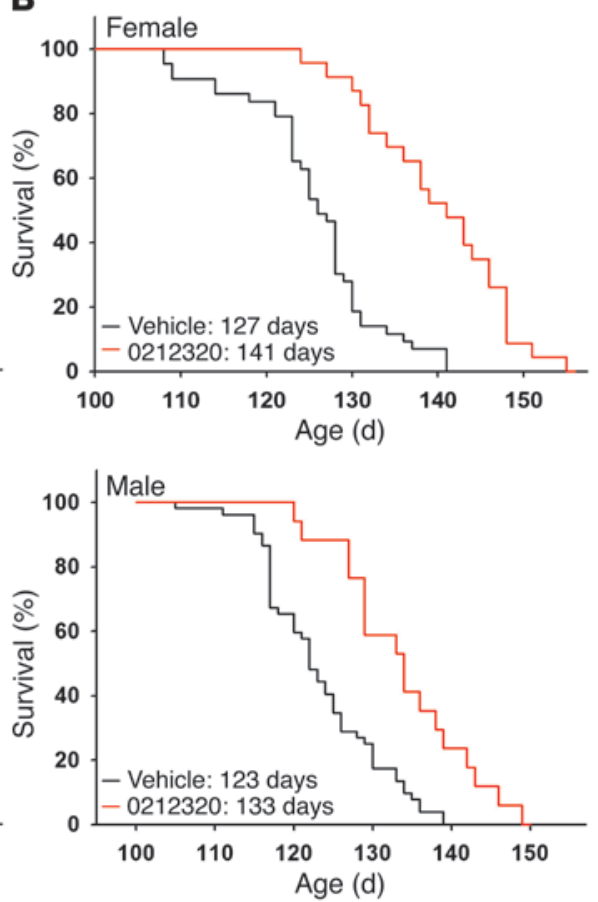
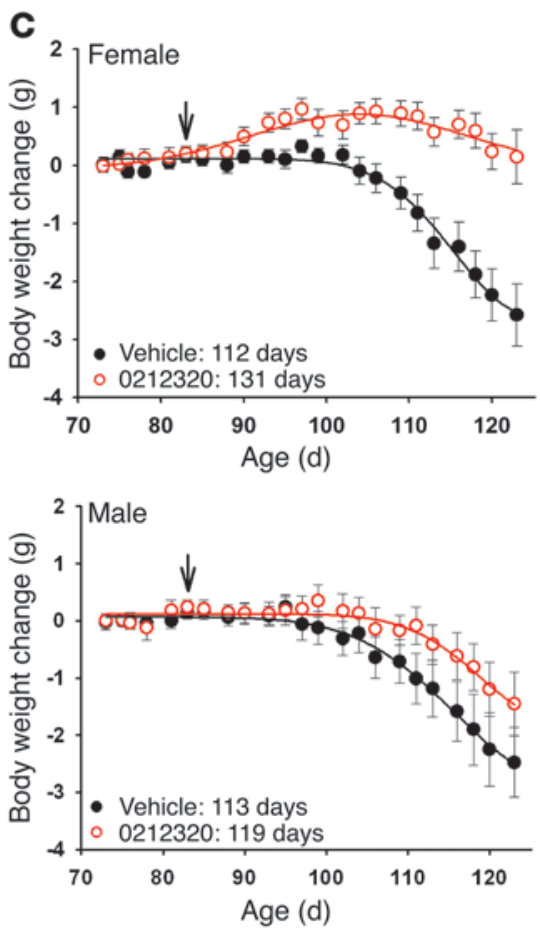

E

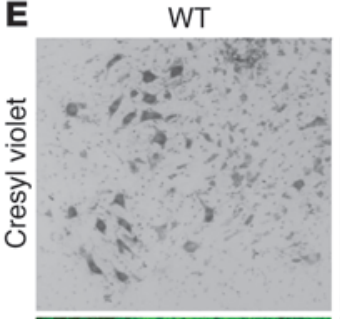

G93A, vehicle

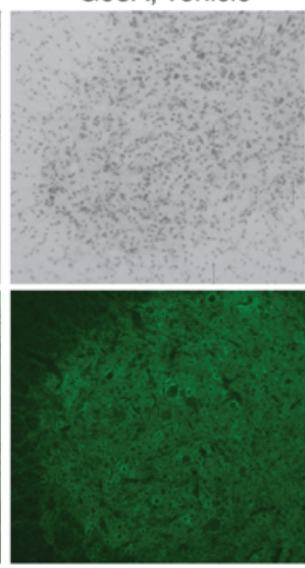

G93A, 0212320

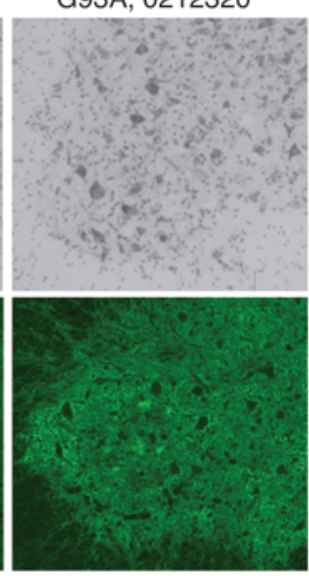

\section{Figure 4}

LDN/OSU-0212320 delays motor function decline and extends lifespan in SOD1(G93A) mice. SOD1(G93A) mice received compound daily (i.p., $40 \mathrm{mg} / \mathrm{kg}$ ) starting at 84 days of age (indicated by arrows in the figure) until death. (A) Motor function decline as assessed by grip strength measurement. Data were analyzed using dynamic fitting nonlinear regression analysis. The average number of days until a $50 \%$ grip strength decline occurred is indicated. (B) Survival results. Data were analyzed using Kaplan-Meier survival analysis. The average number of days of survival is indicated. (C) Body weight change. The average number of days until a 10\% decline in initial body weight occurred is indicated. (D) Representative female mice at 126 days old. (E) Representative images showing cresyl violet staining and EAAT2 immunostaining of the ventral horn region of the spinal cord from 120-day-old female mice. Scale bar: $50 \mu \mathrm{m}$. (F) Western blot results show that LDN/OSU-0212320 restores EAAT2 protein levels. Spinal cords from 120-day-old mice were analyzed. Six animals in each group were analyzed for $\mathbf{E}$ and $\mathbf{F}$.

All other kinases tested showed insignificant activity (Supplemental Table 2). These studies indicate that LDN/OSU-0212320 has high specificity and low side effect/toxicity potential.

Furthermore, the following observations indicate that the effects of LDN/OSU-0212320 on EAAT2 protein expression were not due to a general increase in protein synthesis. First, the total amount of protein in compound-treated cells and mice was not increased compared with that in vehicle-treated cells and mice. Second, we exam- ined the expression of a variety of other proteins in mouse brains: astrocyte-specific proteins including GFAP, glutamine synthetase (GS), and EAAT1 (another astrocytic glutamate transporter subtype); several housekeeping proteins including GAPDH, SOD1, actin, YB-1, and RNA polymerase II; several neuron-specific proteins including synaptophysin, EAAT3 (a neuronal glutamate transporter), EAAT4, PSD95, NMDA receptor subunit NR2B, and vesicular glutamate transporter 1 (VGLUT1); and several signaling proteins including 


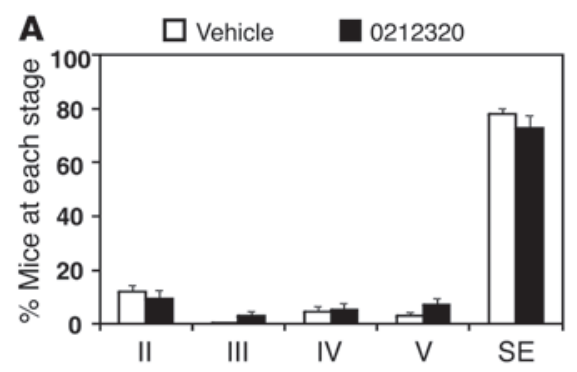

D

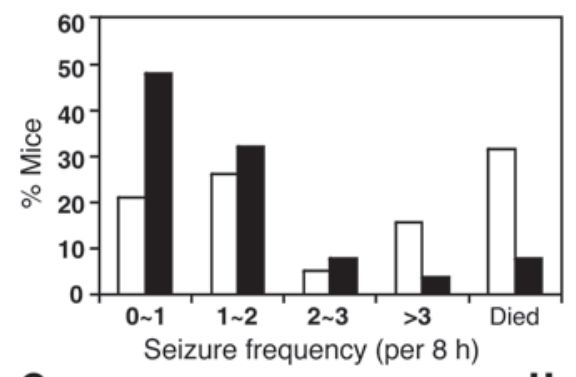

G

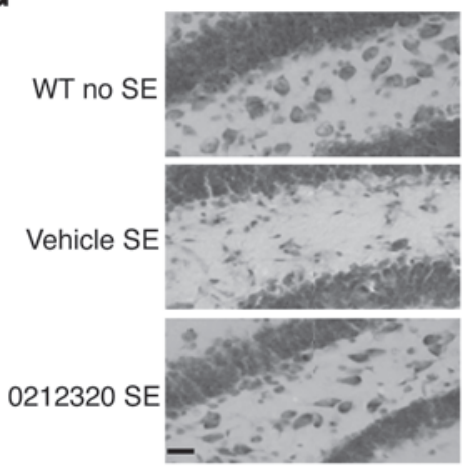

B

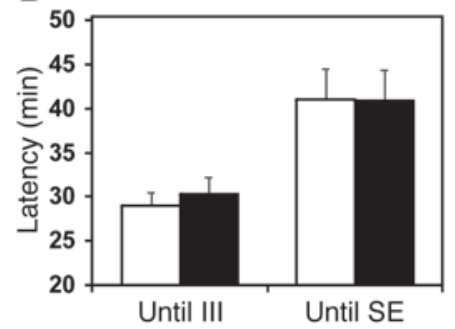

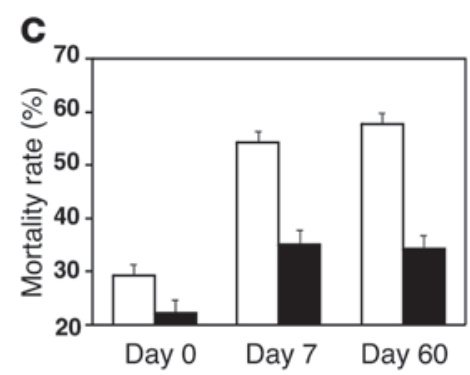

$\mathbf{F}$
E

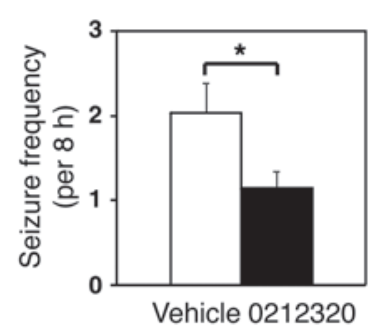

H

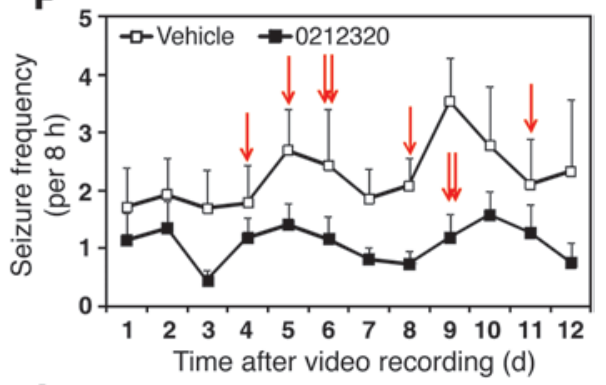

I
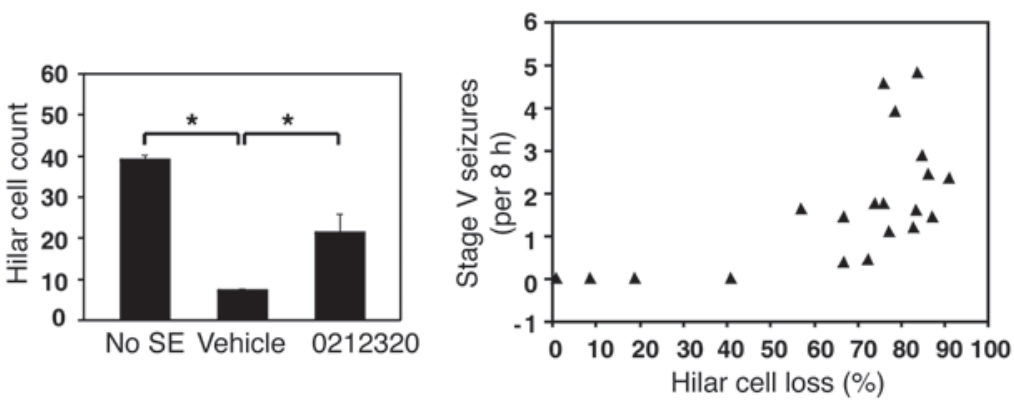

\section{Figure 5}

LDN/OSU-0212320 reduces mortality rate, chronic seizure, and neuronal death following pilocarpine-induced SE. Mice received compound (i.p., $40 \mathrm{mg} / \mathrm{kg}$ ) and were treated with pilocarpine 3 hours later. Mice that reached SE received compound daily, and chronic seizures were recorded 4 weeks after SE for a 2-week period (15 independent experiments in a total of 153 vehicle- and 152 compound-treated mice). (A) Acute seizure severity (maximal seizure activity of each animal within 2 hours after pilocarpine injection). The percentage of mice that reached each seizure stage did not differ between the two groups. (B) Latency (time interval between pilocarpine injection and the indicated stage). Latency did not differ between the two groups. (C) Mortality rate (percentage of mice that died in each experiment, $n=15$ ). A significant decrease was observed in the compound-treated group on days 7 and 60 after SE. (D-F) Spontaneous recurrent seizures. (D) Percentage of mice (vehicle, $n=19$; compound, $n=25$ ) that developed $<1$, 1 2, 2 3, or >3 stage $V$ seizures in an 8-hour period. (E) Average number of stage $V$ seizures in an 8-hour period. (F) Frequency of stage $V$ seizures during each day of recording. Chronic seizure frequency was significantly reduced in compound-treated mice. Arrows indicate that a mouse died on that day. ( $\mathbf{G}$ and $\mathbf{H}$ ) Hippocampal damage. Nine sets of brains 8 weeks after SE were analyzed with cresyl violet staining (15-20 sections/mouse). (G) Representative images of the dentate hilus. Scale bar:20 $\mu \mathrm{m}$. (H) Quantitative analysis of hilar cells. Compound treatment significantly attenuated cell damage. (I) A strong positive correlation was detected between hilar cell loss and chronic seizure frequency. ${ }^{*} P<0.05$.

AKT, PI3K, IKB- $\alpha$, and ERK2. None of the above proteins were induced by LDN/OSU-0212320 (Figure 3G), suggesting that LDN/ OSU-0212320 did not induce global protein synthesis.

LDN/OSU-0212320 slows disease progression and extends the lifespan of SOD1(G93A) mice. Transgenic mice expressing familiar ALS-linked mutant superoxide dismutase (SOD1) have been commonly used for ALS preclinical trials. The SOD1(G93A) mouse, in particular, the high copy number line, has been the most commonly used transgenic mouse model in ALS preclinical trials (20-22). SOD1(G93A) mice exhibit loss of motor neurons and associated motor functions starting at approximately 80 to 90 days of age and die at approximately 4 to 5 months of age. Mice begin to lose EAAT2 protein in the spinal cord at approximately 80 to 90 days of age, and EAAT2 protein levels gradually decrease with disease progression. We previously crossed EAAT2 transgenic mice, which have a 1.5- to 2-fold increase in EAAT2 protein levels, with SOD1(G93A) mice and found that increased EAAT2 expression delayed disease onset, but not the survival of mice (19). Furthermore, Rothstein et al. reported that treating SOD1(G93A) mice with ceftriaxone $(200 \mathrm{mg} / \mathrm{kg})$ markedly ameliorated symptoms and prolonged survival (15).

To determine whether translational activation of EAAT2 was protective in a mouse model of ALS, we evaluated the effects of LDN/OSU-0212320 in the SOD1(G93A) mouse. Several preliminary experiments were conducted. First, to ensure that LDN/ OSU-0212320 could restore EAAT2 protein levels in SOD1(G93A) mice, we administered compound by intrathecal injection into 
A
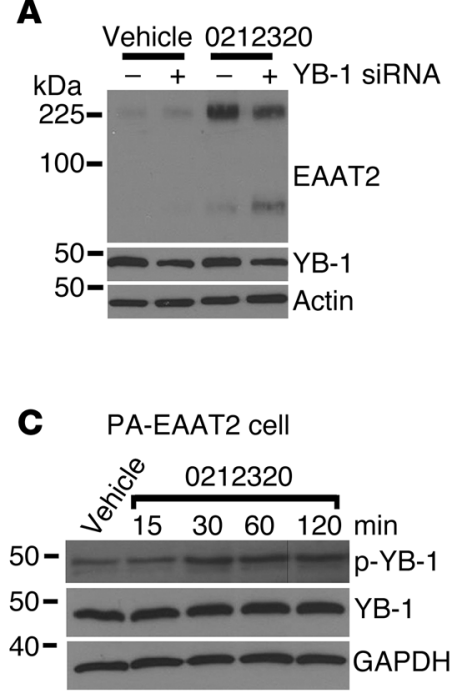

E

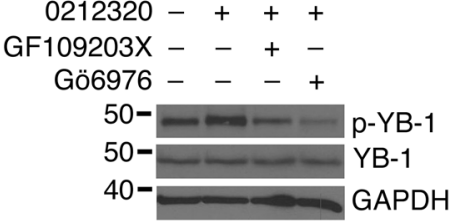

G

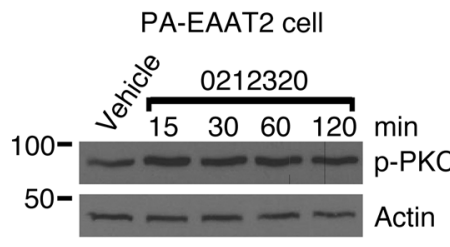

B
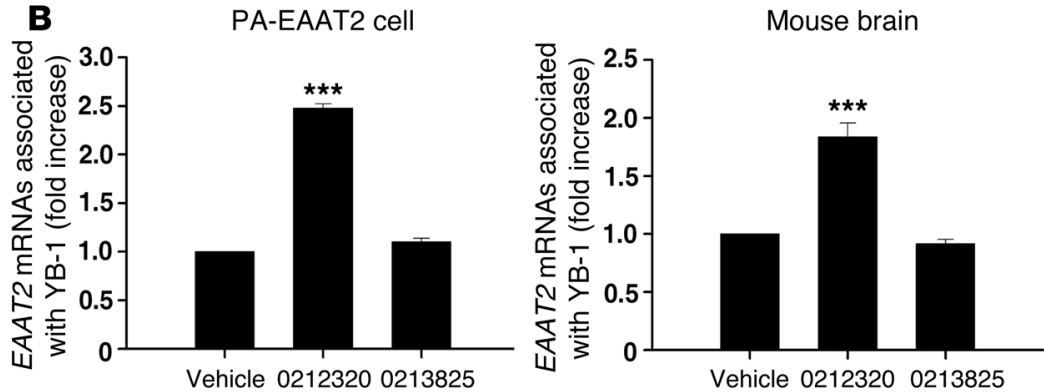

Mouse brain
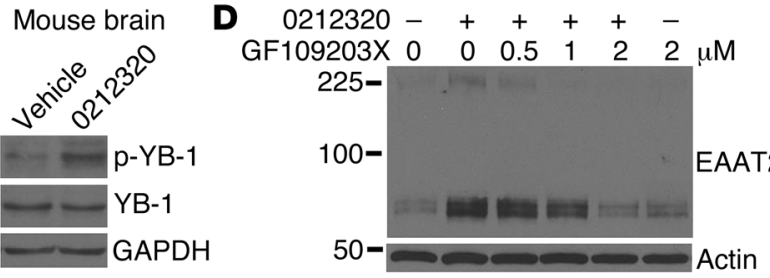

$0212320-++++$ Gö6976 $0 \quad 0 \quad 0.5 \quad 1 \quad 2 \quad 2 \mu M$
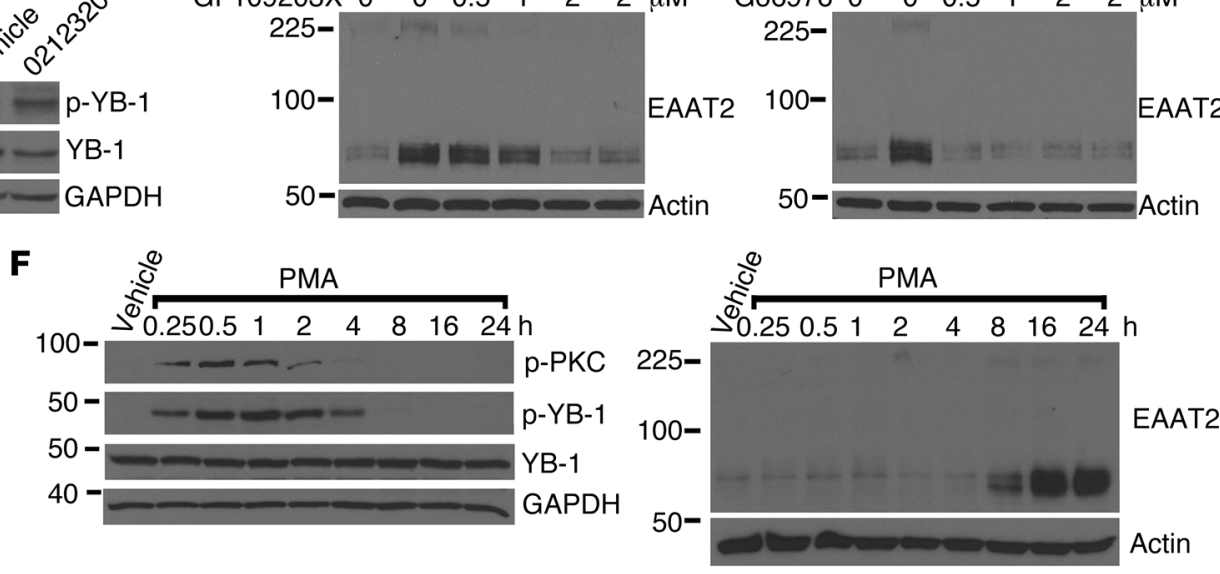

\section{Figure 6}

LDN/OSU-0212320 treatment results in activation of PKC, which subsequently activates YB-1, leading to enhancement of EAAT2 translation. (A) Depletion of YB-1 by siRNA reduced compound-induced EAAT2 expression in PA-EAAT2 cells. Cells were transfected with YB-1 siRNA and were treated 24 hours later with compound $(10 \mu \mathrm{M})$ for 24 hours. Lanes were run on the same gel but were noncontiguous. (B) Increased YB-1EAAT2 mRNA interaction following compound treatment. PA-EAAT2 cells were treated with compound (10 $\mu \mathrm{M})$ for 2 hours and then harvested for immunoprecipitation (anti-YB-1 antibodies). Coimmunoprecipitated EAAT2 mRNAs were quantified by real-time RT-PCR. Mice received a single dose of compound (40 mg/kg, i.p.), and brains were harvested at 2 hours for immunoprecipitation. $n=5$. (C) Compound increased YB-1 phosphorylation (p-YB-1). PA-EAAT2 cells: $10 \mu \mathrm{M}$ compound. Mouse brain: $40 \mathrm{mg} / \mathrm{kg}$, harvested at 1 hour. (D) PKC inhibitors blocked compound-induced EAAT2 expression in PA-EAAT2 cells. Cells were pretreated with inhibitors for 1 hour and then treated with compound (10 $\mu \mathrm{M})$ for 24 hours. (E) PKC inhibitors blocked compound-induced YB-1 phosphorylation in PA-EAAT2 cells. (F) PMA increased YB-1 phosphorylation concomitantly with PKC phosphorylation (p-PKC). Increased EAAT2 protein levels were not observed until 8 hours after treatment. (G) Compound increased PKC phosphorylation. PA-EAAT2 cells: $10 \mu \mathrm{M}$ compound. Mouse brain: $40 \mathrm{mg} / \mathrm{kg}$, harvested at 1 hour. (H) Compounds of different potencies were examined for their effects on PKC activation. p-PKC levels are highly correlated with EAAT2 induction levels. Each data point represents the mean of three experiments. ${ }^{\star \star \star} P<0.001$.

the lumbar region of the spinal cord of 110-day-old mice $(10 \mathrm{mM}$, 3 injections of $5 \mu \mathrm{l} /$ injection/day). The results showed that LDN/ OSU-0212320 was able to restore EAAT2 protein levels and function in the disease stage in SOD1(G93A) mice (data not shown). Second, to select a dose for long-term studies, we tested two doses of LDN/OSU-0212320 (10 and $40 \mathrm{mg} / \mathrm{kg}$ ) by daily i.p. administration ( $n=6$ per group) starting at 84 days of age, when the mice had lost approximately $10 \%$ to $15 \%$ of their motor function (the early symptomatic stage), which may be equivalent to the stage at which ALS patients first visit a doctor. We observed that the dose of $10 \mathrm{mg} / \mathrm{kg}$ had no effect on disease onset (as assessed by motor function decline described below), but extended survival by approximately 11 days. We found that the $40 \mathrm{mg} / \mathrm{kg}$ dose had better effects: delayed motor function decline for approximately 11 days and prolonged survival 
for approximately 12 days. Third, to ensure that $40 \mathrm{mg} / \mathrm{kg}$ is a safe dose for long-term treatment, we treated WT mice at this dose for 2 months. We observed that LDN/OSU-0212320-treated mice $(n=6)$ exhibited behavior similar to that of nontreated mice $(n=6)$. We observed no differences in body weight between compound-treated and nontreated mice. Immunohistochemical characterization of the brains revealed that long-term treatment did not cause neuronal loss (as assessed by cresyl violet staining and nuclear staining), astroglial activation (as assessed by GFAP immunostaining), or microglial activation (as assessed by CD11b immunostaining) (Supplemental Figure 1). We found that LDN/OSU-0212320 did not cause adverse effects after long-term treatment in mice.

We then evaluated the effects of LDN/OSU-0212320 in SOD1(G93A) mice at $40 \mathrm{mg} / \mathrm{kg}$ in detail. We treated the mice with compound from the age of 84 days until death. LDN/OSU-0212320treated (females, $n=23$; males, $n=22$ ) and vehicle-treated (females, $n=18$; males, $n=21$ ) animals were monitored three times per week for motor function by measuring grip strength and were also monitored for survival and body weight. The results showed that in female mice, compound treatment significantly delayed motor function decline by approximately 14 days (50\% grip strength: vehicle, 108 days vs. compound, 122 days) (Figure 4A), prolonged overall survival by approximately 15 days (vehicle, $126.8 \pm 2.4$ days vs. compound, $141.1 \pm 1.5$ days; $P<0.001$, Kaplan-Meier survival analysis with logrank test) (Figure 4B), and delayed body weight decline by approximately 19 days (10\% body weight loss: vehicle, 112 days vs. compound, 131 days) (Figure 4C). These effects were not as significant in male mice, which showed a difference in delayed motor function decline of approximately 12 days (vehicle, 103 days vs. compound, 115 days), a difference in prolonged survival of approximately 10 days (vehicle, $123.4 \pm 2.4$ days vs. compound, $133.2 \pm 2.8$ days; $P=0.013$, Kaplan-Meier survival analysis with log-rank test), and a difference in delayed body weight decline of 6 days (vehicle, 113 days vs. compound, 119 days) (Figure 4, A-C). This difference between the sexes may be due to an earlier onset of disease symptoms in male mice that required earlier treatment in order to be equivalent to the results seen in the females. Figure 4D shows representative female mice at 126 days old. Figure 4, E and F, shows that LDN/OSU-0212320 restores EAAT2 protein levels and partially protects motor neurons from degeneration. These results indicate that LDN/OSU-0212320 has marked protective effects in this ALS disease model.

LDN/OSU-0212320 reduces mortality, spontaneous recurrent seizure, and neuronal death in a pilocarpine-induced status epilepticus mouse model. Several lines of evidence suggest that high extracellular glutamate levels play a crucial role in seizure initiation and propagation (23-28). We previously investigated pilocarpine-induced status epilepticus (SE) in EAAT2 transgenic mice and observed that increased EAAT2 expression significantly prevented SE-induced neuronal death, epileptogenesis, and subsequent recurrent seizures (29). We evaluated the effects of LDN/OSU-0212320 in this epilepsy model by first testing two doses of LDN/OSU-0212320, 20 and $40 \mathrm{mg} / \mathrm{kg}$, in male FVB mice ( $n=16$ per group). In order to evaluate the effects of LDN/OSU-0212320 on early acute seizure activity and later chronic seizure activity, we pretreated mice with compound prior to induction of seizures with pilocarpine. We observed dose-dependent protection against SE-induced neuronal death and recurrent seizures. We have previously demonstrated that a 2 -month administration of $40 \mathrm{mg} / \mathrm{kg}$ LDN/OSU-0212320 did not cause overt toxicity or adverse effects (Supplemental Figure 1). Therefore, this dose was used in the following studies.
Littermate-matched mice received $40 \mathrm{mg} / \mathrm{kg}$ of compound or vehicle by i.p. injection. Three hours later, the mice received pilocarpine to induce seizures and were observed for 2 hours to record acute seizure severity and the latency period. The Racine scale was used to rate seizure severity (stages I, II, III, IV, V, and SE). Fifteen independent experiments ( 20 animals in each experiment) were performed with a total of 152 LDN/OSU-0212320-treated and 153 vehicle-treated male mice. We observed that the percentage of mice that reached each seizure stage (seizure severity) was not significantly different between the compound group and the vehicle group (Figure 5A; $P=0.339$, $\chi^{2}$ test). Latency, which was the time interval between the pilocarpine injection and each seizure stage, was also not significantly different between the two groups (Figure 5B; stage III: $P=0.86$; SE: $P=0.70$; Mann-Whitney rank sum test), suggesting that LDN/OSU-0212320 has no significant effects on early acute seizure activity.

Mice that reached SE and maintained tonic limb extension for at least 60 minutes were used for subsequent studies. We further treated the mice with $40 \mathrm{mg} / \mathrm{kg} /$ day of compound until euthanized for studies. We observed several striking phenomena. The mortality rate after SE is an important parameter for evaluation of antiepileptic effects. As shown in Figure 5C, there was no obvious difference in mortality rates between compound and vehicle groups on the day that SE was induced. However, the mortality rates were significantly decreased in the compound treatment group compared with those in the vehicle treatment group on day 7 after SE (vehicle, 82 of 153 vs. compound, 51 of $152 ; P=0.03, \chi^{2}$ test) and also on day 60 after SE (vehicle, 53 of 90 vs. compound, 33 of $90 ; P=0.005, \chi^{2}$ test).

SE can cause complex molecular, biochemical, and structural changes in the brain, which over time result in the development of spontaneous recurrent seizures. We monitored SE-induced chronic seizures by video recording to determine the frequency and severity of seizure activity. Mice were monitored for 2 weeks ( 8 hours/day) starting 4 weeks after SE, and the average frequency of stage $V$ seizures that occurred in an 8 -hour period in each mouse was quantified. Six of 19 vehicle-treated mice and 2 of 25 compound-treated mice died during the recording period (Figure 5F, arrows). Compound-treated mice developed fewer stage V seizures (Figure 5D). The average seizure (stage $V$ ) frequency in compound-treated mice $(1.14 \pm 0.21)$ was reduced by about half of that observed in vehicle-treated littermates $(2.03 \pm 0.37 ; P=0.025$, Student's $t$ test) (Figure $5 \mathrm{E})$. The repeated measurements of stage $V$ seizure frequency during the recording period were analyzed using generalized linear mixed-effects models with a $\log$ link function. We included a random intercept term to account for the within-subject correlation between repeated measures. There was a significant difference between the compound group and the vehicle group in terms of stage $\mathrm{V}$ seizure occurrence over time (Figure $5 \mathrm{~F}$ ); specifically, LDN/OSU-0212320 treatment tended to reduce the seizure occurrence number by 1.0961 on average $(P=0.012)$.

Finally, intense seizure activity associated with SE causes significant neuronal degeneration in hippocampal regions. We examined hippocampal damage 8 weeks after SE, when chronic seizures had developed. Coronal sections were stained with cresyl violet to identify live cells. We observed SE-induced neuronal death in several hippocampal subregions, including CA1, CA3, and the dentate hilus in both compound- and vehicle-treated brains; however, there was an overall significant reduction of neuronal death in the compound-treated brains. Hilus interneurons were the most vulnerable after SE. Representative images of the dentate hilus are shown in Figure 5G. We counted the live hilar cells under a light microscope. On average, there were about $39.0 \pm 1.2$ neurons per 
section in the hilus of control mice (no SE), while only $7.3 \pm 0.5$ neurons were alive in vehicle-treated mice after SE. We observed a significant increase in neuronal survival $(21.4 \pm 4.6$ neurons per section) in compound-treated mice (Figure $5 \mathrm{H} ; \mathrm{P}<0.05$ ). A strong positive correlation was detected between hilar cell loss and chronic seizure frequency (Figure 5I; correlation coefficient $=0.68$, $P<0.001$, Spearman's rank order correlation test). Taken together, the above studies indicate that LDN/OSU-0212320 is able to significantly reduce SE-induced mortality, spontaneous recurrent seizure, and neuronal death. We did not observe these protective effects in the inactive compound LDN/OSU-0213825, which has a structure similar to that of LDN/OSU-0212320, but cannot increase EAAT2 protein levels (Supplemental Figure 2).

LDN/OSU-0212320 treatment results in activation of $P K C$ and subsequent $Y B-1$ activation, which regulates enhancement of EAAT2 translation. Y-boxbinding protein 1 (YB-1) is a multifunctional protein that binds DNA and RNA and plays an important role in transcriptional and translational regulation $(30,31)$. A recent study indicated that $\mathrm{YB}-1$ interacts with Eaat2 mRNAs in the mouse brain (32). To examine whether YB-1 is involved in compound-activated EAAT2 translation, we performed siRNA knockdown experiments and found that depletion of YB-1 markedly reduced LDN/OSU-0212320-induced EAAT2 expression in PA-EAAT2 cells (Figure 6A). We then examined whether LDN/ OSU-0212320 altered YB-1 and EAAT2 mRNA interaction. YB-1 proteins were immunoprecipitated with anti-YB-1 antibodies, and the coimmunoprecipitated EAAT2 mRNAs were quantified by real-time RT-PCR. The results showed a significant increase in EAAT2 mRNA associated with YB-1 in PA-EAAT2 cells 2 hours after adding LDN/ OSU-0212320, but not in the inactive compound-treated (LDN/ OSU-0213825-treated) cells (Figure 6B). We also found increased YB-1-EAAT2 mRNA interaction in mouse brains 2 hours after i.p. injection of LDN/OSU-0212320 in mice, but not in mice injected with inactive compound (Figure 6B). Furthermore, we found that LDN/OSU-0212320 increased YB-1 phosphorylation at the Ser102 site in PA-EAAT2 cells within 30 minutes of adding compound (Figure 6C). We also observed increased YB-1 phosphorylation in mouse brains within 1 hour of i.p. injection in mice with LDN/OSU0212320 (Figure 6C). The inactive compound (LDN/OSU-0213825) did not increase YB-1 phosphorylation (data not shown).

To identify the signaling pathways involved in compound-activated YB-1 phosphorylation and subsequent EAAT2 translation, we performed inhibition studies in PA-EAAT2 cells. We found that protein kinase C (PKC) inhibitors GF109203X and Gö6976 blocked both EAAT2 induction in a dose-dependent manner (Figure 6D) and YB-1 phosphorylation (Figure 6E). However, GF109203X and Gö6976 are known to inhibit two other kinases, RSK2 and p70S6 kinase (33). RSK2 is regulated by the MEK/ERK pathway, and p70S6 kinase is regulated by the mTOR pathway. We examined whether U-0126, an MEK inhibitor, and rapamycin, an mTOR inhibitor, blocked LDN/OSU-0212320-activated EAAT2 expression. The results showed that neither U-0126 nor rapamycin affected EAAT2 induction (data not shown). We then investigated whether PMA (phorbol-12-myristate-13-acetate), a PKC activating agent, could induce EAAT2 expression. The results showed that PMA increased YB-1 phosphorylation concomitantly with PKC phosphorylation within 30 minutes (Figure 6F). However, increased EAAT2 protein levels were not observed until 8 hours after treatment (Figure 6F), unlike LDN/OSU-0212320, which could induce EAAT2 expression within 2 hours (Figure 1C). These results indicate that other factors induced by the compound are needed for rapid EAAT2 induction.
The PKC family consists of fifteen isozymes. GF109203X inhibits $\mathrm{PKC} \alpha, \beta 1, \delta, \varepsilon$, and $\zeta\left(\mathrm{IC}_{50}=0.008,0.018,0.210,0.132\right.$, and $5.8 \mu \mathrm{M}$, respectively). Gö6976 inhibits $\mathrm{PKC} \alpha$ and $\beta 1\left(\mathrm{IC}_{50}=2.3\right.$ and $6.2 \mathrm{nM}$, respectively), suggesting that either PKC $\alpha$ or $\beta 1$ is involved in the activation process. To determine the isozyme that may be involved, we tested another PKC inhibitor, Gö6983, which inhibits PKC $\alpha, \beta$, $\gamma, \delta$, and $\zeta\left(\mathrm{IC}_{50}=7,7,6,10\right.$, and $60 \mathrm{nM}$, respectively), but not $\beta 1$, and found that this inhibitor did not affect compound-activated YB-1 phosphorylation or EAAT2 expression (data not shown). These results suggest that PKC $\beta 1$ is involved in LDN/OSU0212320 -activated EAAT2 expression. PKC is phosphorylated at three sites in vivo (34). An autophosphorylation on a carboxyl terminus serine residue (e.g., Ser661 in $\beta 1$ ) regulates the PKC's plasma membrane localization (34), where it interacts with diacylglycerol (DAG) and is transformed into a fully active enzyme. To further support the activation of PKC, we used phosphorylated PKC antibodies that detect PKC only when phosphorylated at the carboxyl terminus serine residue (e.g., Ser661 in $\beta 1$ ). The results showed that LDN/OSU-0212320 increased serine phosphorylation within 15 minutes after addition to PA-EAAT2 cells (Figure 6F). We did not observe this increase in the presence of GF109203X or Gö6976 (data not shown). Increased serine phosphorylation was also found in mouse brains within 1 hour after i.p. injection of LDN/OSU0212320 (Figure 6G). The inactive compound LDN/OSU-0213825 did not increase PKC phosphorylation (data not shown). To further confirm that PKC activation is related to EAAT2 induction, we examined serine phosphorylation levels in PA-EAAT2 cells treated with compounds of different potencies. The results showed that phosphorylated PKC levels are highly correlated with EAAT2 induction levels (Figure 6H; correlation coefficient $=0.9, P=0.0015$ ). These results indicate that LDN/OSU-0212320 treatment results in the activation of PKC, which subsequently stimulates YB-1, leading to enhancement of EAAT2 translation.

\section{Discussion}

The results reported here show that increased EAAT2 expression via translational activation by LDN/OSU-0212320 prevents excitotoxicity and provides substantial beneficial effects in ALS and epilepsy animal models. These results also suggest that our novel approach has therapeutic potential for both acute and chronic neurodegenerative diseases.

Memantine and riluzole are currently marketed drugs that have antiexcitotoxic properties. Memantine is a noncompetitive NMDA receptor antagonist and has been shown to have mild clinically relevant effects in moderate-to-severe AD (35). Riluzole inhibits the release of glutamate and also blocks some of the postsynaptic effects of glutamate (36). Riluzole is the only approved pharmacologic treatment for ALS that prolongs survival by about 2 to 3 months (37). Furthermore, ceftriaxone is an FDA-approved $\beta$-lactam antibiotic that can increase EAAT2 expression via the transcriptional activation mechanism $(13,15)$. A large clinical trial was undertaken recently to test ceftriaxone in ALS patients, but was stopped because predetermined criteria for efficacy were not met (38). This disappointing outcome raises a concern about whether increased EAAT2 expression can provide beneficial effects in humans. In our opinion, there are several reasons why this EAAT2 activation approach should not be disregarded. First, it is uncertain whether ceftriaxone is able to effectively increase EAAT2 expression. While there is a body of evidence for ceftriaxone's neuroprotection in vivo that is assumed to be a consequence of 
EAAT2 upregulation, we note that not all studies have confirmed that EAAT2 can be upregulated by ceftriaxone $(39,40)$. Similarly, our study found only marginal upregulation of EAAT2 with ceftriaxone administration to mice (Figure 3F). Second, there is discrepancy in the literature regarding the efficacy of ceftriaxone in SOD1(G93A) mice. The original paper by Rothstein et al. showed that ceftriaxone treatment slowed disease progression and prolonged survival of SOD1(G93A) mice (15). However, Scott et al. retested ceftriaxone in SOD1(G93A) mice using an optimal study design and found no survival benefit (41). Kong et al. also reported no beneficial effects of ceftriaxone in SOD1(G93A) mice (42). Third, it is unknown whether EAAT2 protein levels and glutamate uptake function were restored in the ALS patients who received ceftriaxone in the clinical trial. In our opinion, the question as to whether increased EAAT2 provides beneficial effects in humans remains inadequately answered.

In comparison with ceftriaxone, LDN/OSU-0212320 has several advantages: (a) better physical-chemical properties for the treatment of CNS diseases (LDN/OSU-0212320 has a lower molecular weight, fewer hydrogen bond donors and acceptors, a lower PSA, a higher cLogP value, and fewer rotatable bonds, all of which should result in significantly better brain penetration); (b) better potency (as shown in Figure 3F); (c) capacity to restore EAAT2 protein levels in SOD1(G93A) mice at a more advanced disease stage (as described above); (d) ability to increase EAAT2 levels more quickly, e.g., within 2 hours (Figure $3 \mathrm{~B}$ ) as compared with ceftriaxone, which requires $24-48$ hours (15).

We found that LDN/OSU-0212320 was able to significantly delay motor function decline (females by $\sim 14$ days, males by $\sim 12$ days) and extend the lifespan of SOD1(G93A) mice (females by $\sim 15$ days, males by $\sim 10$ days). This study followed the guidelines established by the ENMC Group (43), which included the use of B6SJL hybrid SOD1(G93A) mice, gender-matched mice, littermate-matched mice, and mice with the same copy number of the SOD1 transgene. Gurney et al. reported that treatment with riluzole at dosages of 24 and $44 \mathrm{mg} / \mathrm{kg}$ beginning at 42 days of age (before the onset of clinical symptoms) extended the survival of SOD1(G93A) mice by about 2 weeks (44). Although many therapeutic agents that show beneficial effects in mutant SOD1 mice are unsuccessful in human trials, SOD1 mice remain the primary preclinical animal model for ALS. These mouse models recapitulate several key features of ALS. Based on the results reported herein, enhancers of EAAT2 translation warrant further investigation as potential therapeutics for ALS. Since ALS is a multifactorial disease and excitotoxicity is a contributing factor, combining LDN/OSU-0212320 with other agents that target different disease mechanisms may also provide greater effects (42).

In the epilepsy study, we pretreated mice with LDN/OSU0212320 prior to induction of seizures, which allowed us to evaluate the effects of LDN/OSU-0212320 at both the acute and chronic stages. The results showed that LDN/OSU-0212320 did not have significant effects on seizure severity at the acute stage, but significantly reduced the mortality rate, recurrent seizures, and neuronal death at the chronic stage. These results indicate that LDN/OSU-0212320 may have antiepileptogenesis function. During epileptogenesis, reactive astrocytes persistently upregulate mGluR5, which could enhance glutamate release (45). In addition, excessive glutamate could be released from astrocytes through volume-sensitive anion channels activated by cell swelling (46) and a $\mathrm{Ca}^{2+}$-dependent exocytosis triggered by inflammatory factors (47).
At present, none of the marketed drugs have been found to attenuate the progression of epileptogenesis. Future studies will apply treatment after SE to further validate the antiepileptogenic effects. Mechanism studies confirmed that LDN/OSU-0212320 did not affect $E A A T 2$ mRNA levels or EAAT2 protein lifespan, but rather activate EAAT2 translational activity. We found that YB-1 contributes to compound-activated EAAT2 translation, although the detailed mechanism of how YB-1 regulates EAAT2 translation remains to be elucidated. LDN/OSU-0212320 treatment resulted in activation of $\mathrm{PKC}$, probably the PKC $\beta 1$ isozyme, which subsequently activates YB-1. Efforts to more clearly define the precise molecular mechanisms of compound-mediated PKC activation, including identifying the compound targets, are currently under investigation.

The continuous optimization of LDN/OSU-0212320, other pyridazine-based derivatives, or other structurally distinct enhancers of EAAT2 translation may lead to improved analogs for human clinical trials. In addition, it is worthwhile to test LDN/OSU0212320 or its analog in other disease models, in which excitotoxicity has been shown to contribute to neurodegeneration such as $\mathrm{AD}$ and cerebral ischemia. Furthermore, understanding the molecular mechanisms underlying the activation of EAAT2 translation by LDN/OSU-0212320 is also very important for advancing this therapeutic approach.

\section{Methods}

Animals. Mice were housed in a 12-hour light/12-hour dark cycle with free access to food and water. All experiments were approved by the IACUC of the Ohio State University and were conducted within the terms of the $\mathrm{NIH}$ guidelines for the care and use of laboratory animals. SOD1(G93A) transgenic mice (B6SJL-Tg [SOD1-G93A] 1Gur with a high copy number of the mutant human SOD1 gene) and FVB mice were purchased from The Jackson Laboratory. Transgenic SOD1(G93A) hemizygotes were maintained by mating transgenic males with B6SJLF1/J hybrid females. Transgenic offspring genotypes were determined by PCR using genomic DNA extracted from tail biopsies.

PA-EAAT2 cell studies. PA-EAAT2 cells were grown in DMEM (Invitrogen) supplemented with $10 \% \mathrm{FBS}, 700 \mu \mathrm{g} / \mathrm{ml}$ geneticin (Gibco) and $100 \mu \mathrm{g} / \mathrm{ml}$ penicillin-streptomycin (Sigma-Aldrich). Cells were maintained at $37^{\circ} \mathrm{C}$ in the presence of $5 \% \mathrm{CO}_{2}$. To evaluate compound effects, cells were cultured in DMEM and treated with compound for the indicated times (Figure 1 and Figure 6) and then harvested for analysis. Western blotting $(16),\left[{ }^{3} \mathrm{H}\right]$ glutamate uptake assays (16), immunofluorescence staining (16), real-time RT-PCR (16), pulsechase analysis (14), polyribosome analysis (48), and subcellular fractionation analysis (49) were performed as described in our previous studies.

Primary dissociated culture studies. Primary dissociated neuron and astrocyte mixed cultures were prepared from newborn (12- to 24-hour-old) FVB WT pups as previously described (50). These cultures were maintained in DMEM and supplemented with $0.5 \% \mathrm{FBS}, 100 \mu \mathrm{g} / \mathrm{ml}$ penicillin-streptomycin, and 1X B-27 (Invitrogen) until use. To evaluate compound effects, cells were cultured in DMEM and treated with compound and/or $100 \mu \mathrm{M}$ glutamate for the indicated times (Figure 2) and then harvested for analysis. Western blotting, $\left[{ }^{3} \mathrm{H}\right]$ glutamate uptake assays, immunofluorescence staining, and LDH assays were performed as previously described $(16,19)$. For the immunofluorescence staining study, images were obtained using a Zeiss Axioskop 2 upright microscope and AxioVision software. The MAP2positive cells with unambiguous neuronal morphology were counted using images at $\times 200$ magnification. Ten random fields per well were counted to achieve an average MAP2-positive neuron number $/ \mathrm{mm}^{2}$ per treatment in every experiment that was repeated five times. The MAP2-positive neuron count $/ \mathrm{mm}^{2}$ in the no-treatment control was considered $100 \%$. 
WT mouse studies. WT adult $\mathrm{C} 57 \mathrm{Bl} / 6$ mice received i.p. administration of LDN/OSU-0212320 at the indicated doses (Figure 3) in $500 \mu \mathrm{l}$ of $1 \%$ DMSO/1\% polyethylene glycol 400/0.2\% Tween 80/10\% hydroxypropyl$\beta$-cyclodextrin/saline (or the indicated formulation) at the indicated time points (Figure 3). Tissue was then harvested either fresh or perfused with $0.9 \% \mathrm{NaCl}$ followed by $4 \%$ PFA in $0.1 \mathrm{M}$ phosphate buffer (PB) for analysis. Western blotting, $\left[{ }^{3} \mathrm{H}\right]$ glutamate uptake assays, cresyl violet staining, immunofluorescence staining, and real-time RT-PCR were performed as previously described $(19,29)$. Pharmacokinetic evaluation of LDN/OSU0212320 was performed using freshly isolated brain tissue. Briefly, after a single i.p. administration $(3 \mathrm{mg} / \mathrm{kg}$ in $2 \%$ hydroxypropyl- $\beta$-cyclodextrin/ $\mathrm{PBS})$ to male $\mathrm{C} 57 \mathrm{Bl} / 6$ mice, plasma and brain concentrations were determined at $0.25,0.5,1,2$, and 8 hours by LC-MS/MS. The plasma half-life was estimated, and the average brain/plasma ratios were tested at all time points. These studies were performed by Absorption Systems.

SOD1(G93A) mouse studies. We followed the guidelines established by the ENMC Group (42). Mice received LDN/OSU-0212320 at the indicated doses (Figure 4) in $500 \mu \mathrm{l}$ of $1 \% \mathrm{DMSO} / 1 \%$ polyethylene glycol 400/0.2\% Tween $80 / 10 \%$ hydroxypropyl- $\beta$-cyclodextrin/saline starting at 84 days of age ( 6 times/week, at the same time each day) until death or until brain tissues were harvested at 120 days of age for analysis. Western blotting, cresyl violet staining, and immunofluorescence staining were performed as previously described (51). Motor function was determined by measuring peak force using a digital grip dynamometer outfitted with a hind limb pullbar assembly (Columbus Instruments). Measurements started at 70 days of age (3 times/week, on the same day of each week and at the same time on each scheduled day) until the mouse lost all strength. Six repetitions were conducted, and the average was determined for each point. The examiners were blinded to the treatment given to the mice. Food and water were placed on the cage floor when mice began to show motor deficits. Mice were euthanized when they were unable to roll over within 30 seconds after being placed on their sides. This time point was recorded as the time of death and as survival in days.

Epilepsy studies. Eight- to 10-week-old male FVB mice (22-32 g) received i.p. injections of LDN/OSU-0212320 (at the indicated dose in the formulation described above) 3 hours prior to the induction of seizures with pilocarpine (290 mg/kg i.p.; Sigma-Aldrich). To reduce peripheral cholinergic effects without interfering with the development of SE and chronic seizures, mice received scopolamine methyl nitrate $(1.5 \mathrm{mg} / \mathrm{kg}$; Sigma-Aldrich) $30 \mathrm{~min}$ utes prior to the pilocarpine injection. The control animals were injected with scopolamine followed by saline, instead of pilocarpine. Mice were then observed for 2 hours to record the acute seizure severity and the latency period. The Racine scale (52) was used to rate seizure severity as previously described (29). Mice that reached SE and maintained tonic limb extension for at least 60 minutes were kept and treated with compound daily for chronic seizure and pathological studies. The SE mice were fed with food soaked in a high-sucrose (10\%) saline solution to help recovery in the first 3 days after SE. Vehicle-treated SE mice and compound-treated SE littermates were carefully matched based on body weight, seizure latency, seizure severity, and post-SE sickness. Therefore, the nature of the SE (i.e., seizure length and severity) in those mice was comparable. Chronic seizure activity was recorded by webcam (Logitech) and reviewed using Windows Live Movie Maker. Daylight periods $(9 \mathrm{am}-5 \mathrm{pm})$ were selected for observations, because the previous literature indicates a higher frequency of spontaneous recurrent seizures during the day $(29,53)$. Eight weeks after SE, brain tissues were harvested from SE mice for cresyl violet staining (29). Neurons that were stained with cresyl violet and that possessed a prominent nucleolus were counted under a light microscope at $\times 200$ magnification. Six animals per group were analyzed. The correlation between hilar cell loss and chronic seizure frequency in SE mice was examined as previously described (29).
Antibodies. The following primary antibodies were used in this study: purified rabbit anti-EAAT2 pAb (1:2,000) (16); rabbit anti-EAAT3 pAb (1:300) (49); rabbit anti-EAAT4 pAb (1:500) (49); rabbit anti-EAAT1 pAb (1:1,000; Abcam); rabbit antiglutamine synthetase pAb (1:400; Santa Cruz Biotechnology Inc.); rabbit anti-GFAP pAb (1:1,000; Promega); mouse antiGAPDH mAb (1:3,000, Santa Cruz Biotechnology Inc.); rabbit anti-AKT $\mathrm{mAb}$ (1:3,000; Cell Signaling Technology); goat anti-actin pAb (1:3,000; Santa Cruz Biotechnology Inc.); mouse anti-actin mAb (1:5,000; Millipore); mouse anti-MAP2 mAb (1:500; NeoMarker); rat anti-CD11b mAb (1:1,000; Chemicon International Inc.); mouse anti-VGLUT1 mAb (1:1,000; Millipore); and rabbit anti-NR2B pAb (1:1,000; Millipore).

Profiling and kinase studies. Profiling was performed by Caliper LifeSciences (now owned by PerkinElmer) in their General SEP I screening program to identify possible side effects from the compound. Seventy-one targets were tested in duplicate at $10 \mu \mathrm{M}$ including 30 neurotransmitter-related receptors, 2 steroids, 6 ion channels, including HERG binding, 1 second messenger, 3 prostaglandins, 4 growth factors/hormones, 13 brain/gut peptides, 5 enzymes, 7 cytochrome P450 isoenzymes (CYPs). For the detailed list, see Supplemental Table 1. Kinase profiling was performed by Nanosyn. Compound was tested at 1 and $10 \mu \mathrm{M}$ for 230 kinases. The complete list of kinases is provided in Supplemental Table 3.

Statistics. The continuous quantitative data in this study were expressed as the mean \pm SEM. $\chi^{2}$ tests were used to examine the distribution difference between vehicle- and compound-treated mice (54). The survival rates of SOD1(G93A) mice were compared between vehicle- and compound-treated groups by Kaplan-Meier survival analysis with a log-rank test. The repeated measures seizure data were analyzed using generalized linear mixed-effects models with a log link function. The correlation between hilar cell loss and chronic seizure frequency in SE mice was examined by the Spearman's rank order correlation test. For multiple group comparisons, data were analyzed using 1-way ANOVA, with all pairwise multiple comparison procedures (Holm-Sidak method) when the data passed the normality and equal variance tests. Otherwise, data were analyzed using a Kruskal-Wallis test, with all pairwise multiple comparison procedures (Tukey's test or Dunn's method). For comparisons between two groups, a 2-tailed Student's $t$ test was performed when the normality assumptions were not violated. Otherwise, a nonparametric Mann-Whitney rank sum test was performed. Values were considered significant at $P<0.05$.

Study approval. All experiments were approved by the IACUC of the Ohio State University and were conducted within the terms of the NIH guidelines for the care and use of laboratory animals.

\section{Acknowledgments}

This work was supported by grants from the NIH/NINDS (R01NS064275 and U01NS074601), the Thome Memorial Foundation, the Alzheimer's Association, and the Harvard NeuroDiscovery Center. We thank Xin He (University of Maryland, College Park, Maryland, USA) for his assistance with statistical analysis and data interpretation; Jeffrey D. Rothstein (The Johns Hopkins University, Baltimore, Maryland, USA) for his advice on the present study; and Karl Obrietan (The Ohio State University, Columbus, Ohio, USA) for his advice on the epilepsy model.

Received for publication August 6, 2012, and accepted in revised form November 27, 2013.

Address correspondence to: Chien-liang Glenn Lin, Department of Neuroscience, The Ohio State University, 333 West 10th Avenue, Columbus, Ohio 43210, USA. Phone: 614.688.5433; Fax: 614.688.8742; E-mail: lin.492@osu.edu. 
1. Choi DW. Ionic dependence of glutamate neurotoxicity. J Neurosci. 1987;7(2):369-379.

2. Chaudhry FA, Lehre KP, van Lookeren Campagne M, Ottersen OP, Danbolt NC, Storm-Mathisen J. Glutamate transporters in glial plasma membranes: highly differentiated localizations revealed by quantitative ultrastructural immunocytochemistry. Neuron. 1995;15(3):711-720.

3. Rothstein JD, et al. Knockout of glutamate transporters reveals a major role for astroglial transport in excitotoxicity and clearance of glutamate. Neuron. 1996;16(3):675-686.

4. Lin CG, Kong Q, Cuny GD, Glicksman MA. Glutamate transporter EAAT2: a new target for the treatment of neurodegenerative diseases. Future Med Chem. 2012;4(13):1689-1700.

5. Rothstein JD, Van Kammen M, Levey AI, Martin LJ, Kuncl RW. Selective loss of glial glutamate transporter GLT-1 in amyotrophic lateral sclerosis. Ann Neurol. 1995;38(1):73-84.

6. Li S, Mallory M, Alford M, Tanaka S, Masliah E. Glutamate transporter alterations in Alzheimer disease are possibly associated with abnormal APP expression. J Neuropathol Exp Neurol. 1997; 56(8):901-911.

7. Jacob CP, et al. Alterations in expression of glutamatergic transporters and receptors in sporadic Alzheimer's disease. J Alzheimers Dis. 2007; 11(1):97-116.

8. Acharya MM, Hattiangady B, Shetty AK. Progress in neuroprotective strategies for preventing epilepsy. Prog Neurobiol. 2008;84(4):363-404.

9. Yi JH, Hazell AS. Excitotoxic mechanisms and the role of astrocytic glutamate transporters in traumatic brain injury. Neurochem Int. 2006;48(5):394-403.

10. Hazell AS. Excitotoxic mechanisms in stroke: an update of concepts and treatment strategies. Newrochem Int. 2007;50(7-8):941-953.

11. Zelenaia O, et al. Epidermal growth factor receptor agonists increase expression of glutamate transporter GLT-1 in astrocytes through pathways dependent on phosphatidylinositol 3-kinase and transcription factor NF-kappaB. Mol Pharmacol. 2000;57(4):667-678.

12. Figiel M, Maucher T, Rozyczka J, Bayatti N, Engele J. Regulation of glial glutamate transporter expression by growth factors. Exp Neurol. 2003; 183(1):124-135.

13. Lee SG, Su ZZ, Emdad L, et al. Mechanism of ceftriaxone induction of excitatory amino acid transporter-2 expression and glutamate uptake in primary human astrocytes. J Biol Chem. 2008; 283(19):13116-13123.

14. Tian G, et al. Translational control of glial glutamate transporter EAAT2 expression. J Biol Chem. 2007; 282(3):1727-1737.

15. Rothstein JD, et al. $\beta$-Lactam antibiotics offer neuroprotection by increasing glutamate transporter expression. Nature. 2005;433(7021):73-77.

16. Colton $\mathrm{CK}$, et al. Identification of translational activators of glial glutamate transporter EAAT2 through cell-based high-throughput screening: an approach to prevent excitotoxicity. J Biomol Screen. 2010;15(6):653-662.

17. Bristol LA, Rothstein JD. Glutamate transporter gene expression in amyotrophic lateral sclerosis motor cortex. Ann Neurol. 1996;39(5):676-679.

18. Xing X, et al. Structure-activity relationship study of pyridazine derivatives as glutamate transporter EAAT2 activators. Bioorg Med Chem Lett. 2011;
21(19):5774-5777.

19. Guo H, et al. Increased expression of the glial glutamate transporter EAAT2 modulates excitotoxicity and delays the onset but not the outcome of ALS in mice. Hum Mol Genet. 2003;12(19):2519-2532.

20. Gurney ME, et al. Motor neuron degeneration in mice that express a human $\mathrm{Cu}, \mathrm{Zn}$ superoxide dismutase mutation. Science. 1994;264(5166):1772-1775.

21. Chiu AY, et al. Age-dependent penetrance of disease in a transgenic mouse model of familial amyotrophic lateral sclerosis. Mol Cell Neurosci. 1995; 6(4):349-362

22. Gurney ME. The use of transgenic mouse models of amyotrophic lateral sclerosis in preclinical drug studies. J Neurol Sci. 1997;152(suppl 1):S67-S73.

23. During MJ, Spencer DD. Extracellular hippocampal glutamate and spontaneous seizure in the conscious human brain. Lancet. 1993; 341(8861):1607-1610.

24. Cavus I, et al. Extracellular metabolites in the cortex and hippocampus of epileptic patients. Ann Neurol. 2005;57(2):226-235.

25. Tian GF, et al. An astrocytic basis of epilepsy. Nat Med. 2005;11(9):973-981.

26. Binder DK, Steinhauser C. Functional changes in astroglial cells in epilepsy. Glia. 2006;54(5):358-368.

27. Wetherington J, Serrano G, Dingledine R. Astrocytes in the epileptic brain. Neuron. 2008;58(2):168-178.

28. Benarroch EE. Astrocyte-neuron interactions: implications for epilepsy. Neurology. 2009; 73(16):1323-1327.

29. Kong Q, Takahashi K, Schulte D, Stouffer N, Lin $\mathrm{Y}$, Lin CL. Increased glial glutamate transporter EAAT2 expression reduces epileptogenic processes following pilocarpine-induced status epilepticus. Neurobiol Dis. 2012;47(2):145-154.

30. Evdokimova V, Ovchinnikov LP, Sorensen PH. Y-box binding protein 1: providing a new angle on translational regulation. Cell Cycle. 2006; 5(11):1143-1147.

31. Kohno K, Izumi H, Uchiumi T, Ashizuka M, Kuwano $\mathrm{M}$. The pleiotropic functions of the Y-box-binding protein, YB-1. Bioessays. 2003;25(7):691-698.

32. Tanaka T, Ohashi S, Funakoshi T, Kobayashi S YB-1 binds to GluR2 mRNA and CaM1 mRNA in the brain and regulates their translational levels in an activity-dependent manner. Cell Mol Neurobiol. 2010;30(7):1089-1100.

33. Davies SP, Reddy H, Caivano M, Cohen P. Specificity and mechanism of action of some commonly used protein kinase inhibitors. Biochem J. 2000; 351(pt 1):95-105.

34. Keranen LM, Dutil EM, Newton AC. Protein kinase $\mathrm{C}$ is regulated in vivo by three functionally distinct phosphorylations. Curr Biol. 1995;5(12):1394-1403.

35. van Marum RJ. Update on the use of memantine in Alzheimer's disease. Neuropsychiatr Dis Treat. 2009; 5:237-247.

36. Cheah BC, Vucic S, Krishnan AV, Kiernan MC. Riluzole, neuroprotection and amyotrophic lateral sclerosis. Curr Med Chem. 2010;17(18):1942-1199.

37. Miller RG, Mitchell JD, Moore DH. Riluzole for amyotrophic lateral sclerosis (ALS)/motor neuron disease (MND). Cochrane Database Syst Rev. 2012; 3:CD001447.

38. Northeast Amyotrophic Lateral Sclerosis Consortium. Statement on the Clinical Trial of Cetriaxone. Clinical ALS Trials information Web site. http://www.nealsconsortium.org/news_ceftriaxone_announcement.php. Updated August 8, 2012.
Accessed July 14, 2013.

39. Melzer N, et al. A B-Lactam antibiotic dampens excitotoxic inflammatory CNS damage in a mouse model of multiple sclerosis. PLoS One. 2008; 3(9):e3149.

40. Carbone M, Duty S, Rattray M. Riluzole elevates GLT-1 activity and levels in striatal astrocytes. Neurochem Int. 2012;60(1):31-38.

41. Scott S, et al. Design, power, and interpretation of studies in the standard murine model of ALS. Amyotroph Lateral Scler. 2008;9(1):4-15.

42. Kong Q, Carothers S, Chang Y, Lin CG. The importance of preclinical trial timing - a potential reason for the disconnect between mouse studies and human clinical trials in ALS. CNS Neurosci Ther. 2012;18(9):791-793.

43. Ludolph AC, et al. Guidelines for the preclinical in vivo evaluation of pharmacological active drugs for ALS/MND: report on the 142nd ENMC international workshop. Amyotroph Lateral Scler. 2007; 8(4):217-223

44. Gurney ME, Fleck TJ, Himes CS, Hall ED. Riluzole preserves motor function in a transgenic model of familial amyotrophic lateral sclerosis. Neurology. 1998;50(1):62-66.

45. Notenboom RG, et al. Up-regulation of hippocampal metabotropic glutamate receptor 5 in temporal lobe epilepsy patients. Brain. 2006; 129(pt 1):96-107.

46. Liu HT, Tashmukhamedov BA, Inoue H, Okada Y, Sabirov RZ. Roles of two types of anion channels in glutamate release from mouse astrocytes under ischemic or osmotic stress. Glia. 2006;54(5):343-357.

47. Domercq M, Brambilla L, Pilati E, Marchaland J, Volterra A, Bezzi P. P2Y1 receptor-evoked glutamate exocytosis from astrocytes: control by tumor necrosis factor-alpha and prostaglandins. $J$ Biol Chem. 2006;281(41):30684-30696.

48. Shan X, Chang Y, Lin CL. Messenger RNA oxidation is an early event preceding cell death and causes reduced protein expression. FASEB J. 2007; 21(11):2753-2764.

49. Butchbach ME, Tian G, Guo H, Lin CL. Association of excitatory amino acid transporters, especially EAAT2, with cholesterol-rich lipid raft microdomains: importance for excitatory amino acid transporter localization and function. J Biol Chem. 2004;279(33):34388-34396.

50. Tian G, Kong Q, Lai L, Ray-Chaudhury A, Lin CL. Increased expression of cholesterol 24S-hydroxylase results in disruption of glial glutamate transporter EAAT2 association with lipid rafts: a potential role in Alzheimer's disease. J Neurochem. 2010; 113(4):978-989.

51. Chang Y, et al. Messenger RNA oxidation occurs early in disease pathogenesis and promotes motor neuron degeneration in ALS. PLoS One. 2008; 3(8):e2849.

52. Racine RJ. Modification of seizure activity by electrical stimulation. II. Motor seizure. Electroencepha$\log$ Clin Neurophysiol. 1972;32(3):281-294.

53. Shapiro LA, Figueroa-Aragon S, Ribak CE. Newly generated granule cells show rapid neuroplastic changes in the adult rat dentate gyrus during the first five days following pilocarpine-induced seizures. Eur J Neurosci. 2007;26(3):583-592.

54. Pitsch J, Schoch S, Gueler N, Flor PJ, van der Putten H, Becker AJ. Functional role of mGluR1 and mGluR4 in pilocarpine-induced temporal lobe epilepsy. Neurobiol Dis. 2007;26(3):623-633. 